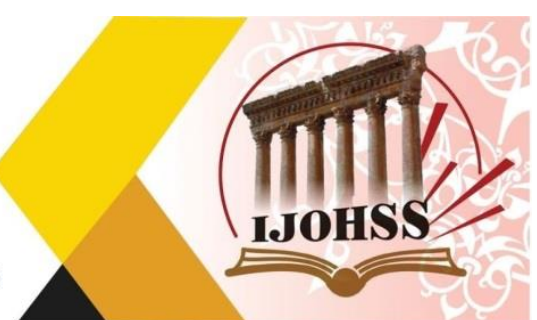

\title{
فاعلية السياسة النقدية في تنشيط الناتج المحلي الاجمالي في العراق خلال المدة 2004 - 2018
}

\author{
المدرس المساعد

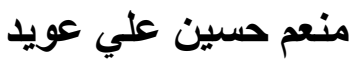 \\ قسم ادارة الاعمال ـ كلية النسور الجامعة عوبل

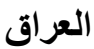 \\ ايميل : monem_hussain@yahoo.com
}

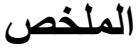

يهدف البحث الى توضيح الدور الذي تلعبة السياسة النقدية في تنشيط الناتج المحلي الاجمالي في العراق، من

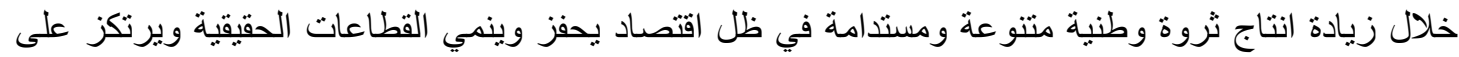
قطاع النفط، عن طريق ادارة الاقتصاد الكلي بالثنكل الذي يعزز من قوة النمو المتوازن للقطاعات الاقتصادية

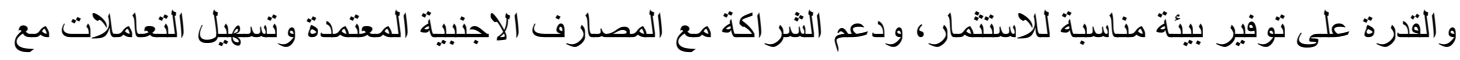
المؤسسات النقدية المحلية وحل المشاكل و العقبات التي تواجه الثركات و الهيئات العاملة في البلد، اذ اوضحت نتائج التحليل القياسي ان متغيرات السياسة النقدية ذات تاثثر معنوي في الناتج المحلي الاجمالي من خلال قيمة (R2=0.96) و (R2=0.94)

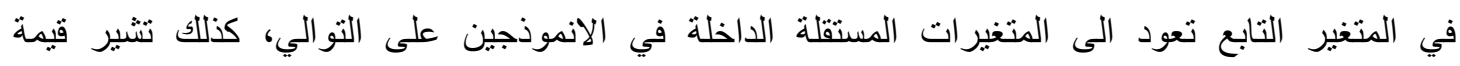

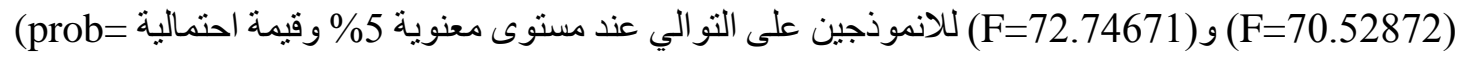
(0.000 الى وجود تكامل مشترك بين المتغيرات الداخلة في الانموذجين مما يعني وجود علاقة توازنية طويلة الاجل، كما اوضحت قيمة (DW=1.387419) و (DW= 1.257052) للانموذجين على التو الي بانة لا توجد مشكلة الارتباط الذاتي لقيم المتغير العشوائي.

الكلمات المفتاحية: السياسة النقدية، عرض النقد، حوالات الخزينة المركزية، الناتج المحلي الاجمالي، ARDL. 


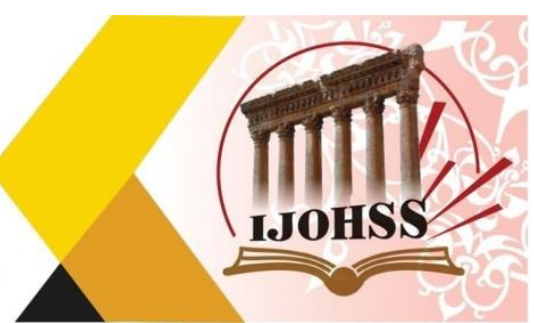

\title{
The Effectiveness of Monetary Policy in Stimulating Gross Domestic Product in Iraq During The Period 2004 - 2018
}

\author{
Assistant Lecturer \\ Monem Hussain Ali Awed \\ Business Administration Department \\ Al-Nisour University College \\ Iraq \\ Email : monem_hussain@yahoo.com
}

\begin{abstract}
The research aims to clarify the role that monetary policy plays in stimulating gross domestic product in Iraq, by increasing the production of a diversified and sustainable national wealth in light of an economy that stimulates and develops the real sectors and focuses on the oil sector, by managing the macro economy in a manner that enhances the strength of balanced growth of sectors Economic capacity and the ability to provide a suitable environment for investment, support the partnership with accredited foreign banks, facilitate transactions with local monetary institutions, and solve problems and obstacles facing companies and agencies operating in the country, as the results of the standard analysis indicated that the policy variables A potency with a significant effect on the gross domestic product through the value of $\left(\mathrm{R}^{2}=0.96\right)$ and $\left(\mathrm{R}^{2}=0.94\right)$, respectively) in the two models, which means that $(96 \%$ and $94 \%)$ of the changes in the dependent variable are due to the independent variables included in the two models, respectively, as well. The value of $(\mathrm{F}=70.52872)$ and $(\mathrm{F}=72.74671)$ for the two models, respectively, at the level of significance of $5 \%$ and the probability value (prob 0.000) indicates that there is a common complementarity between the variables included in the two models, which means a long-term balanced relationship, as indicated by the value of $(\mathrm{DW}=1.387419)$ and

$(\mathrm{DW}=1.257052)$ for the two models, respectively, that there is no problem of self-correlation of random variable values.
\end{abstract}

Keywords: Monetary Policy, Money Supply, Central Treasury Transfers, Gross Domestic Product, ARDL. 


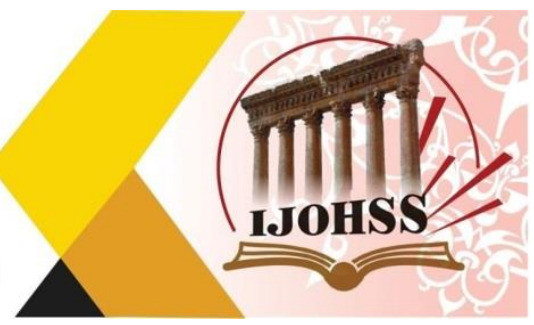

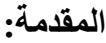

تعد السياسة النقدية في العراق واحدة من اهم السياسات الاقتصادية الاكثر فاعلية في دعم النشاط

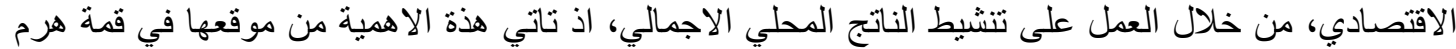

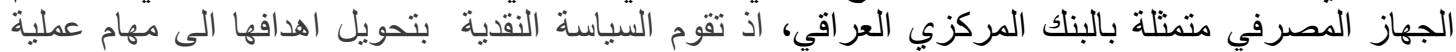

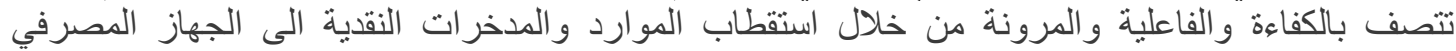

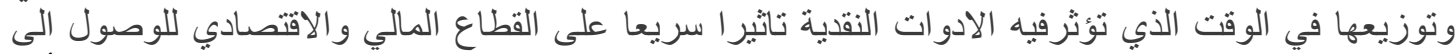

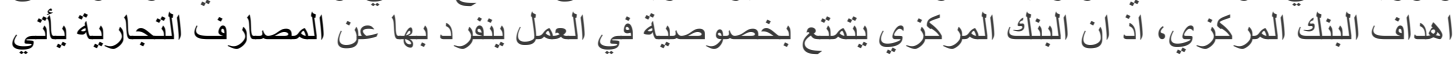

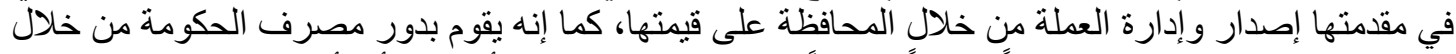

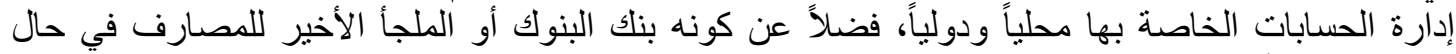

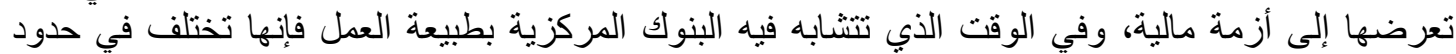

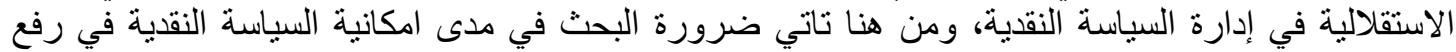
مسنوى النشاط الاقتصادي عن طريق تنشيط الناتج المحلي الاجمالي في العراق.

\section{المبحث الاول}

اولا : منهجية الدراسة المبة

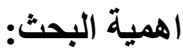

تبرز اهمية البحث من خلال تفعيل دور السياسة النقدية في العمل على تنشيط الناتج المحلي الاجمالي في

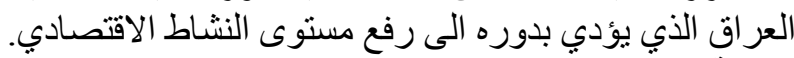

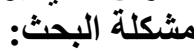

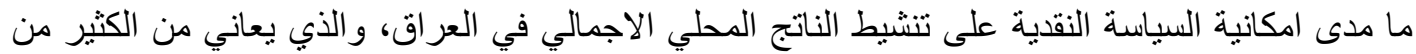

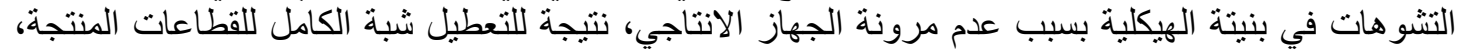
و اعتماد العر اق وبشكل كبير على الاير ادات النفطية فضلا عن اعتمادة على الواردات في تمويل الطلب المحلي.

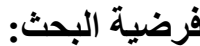

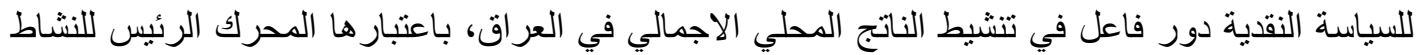

الاقتصادي من خلال التحكم في حجم الكتلة النقدية عن طريق التوسي التيع في دعم المشاريع الاستثمارية المنتجة.

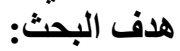

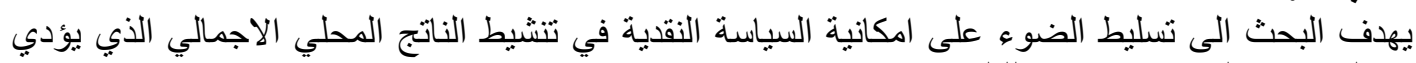
بدوره الى زيادة النمو الاقتصادي للبلدا.

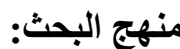
اعنمد البحث على المنهج الوصفي فيما يتعلق بالاطار النظري، واعتمد على المنهج التحليلي فيما يخص

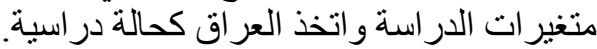

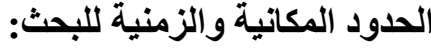

يتخذ البحث حدوده المكانية من الاقتصاد العر اقي، والزمنية خلال المدة (2004_2018).

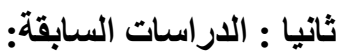

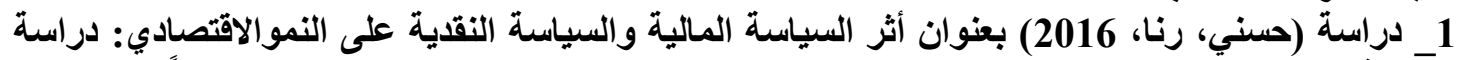

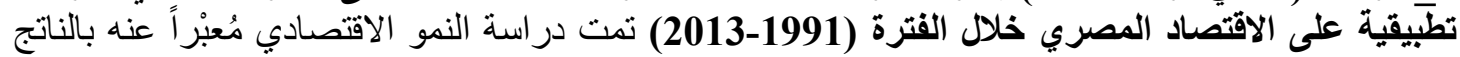

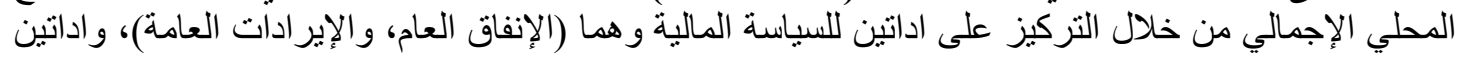

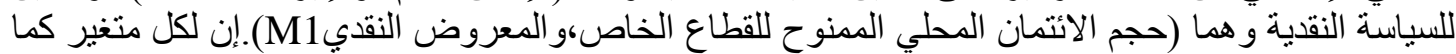

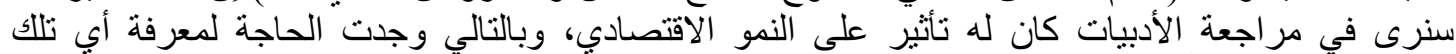

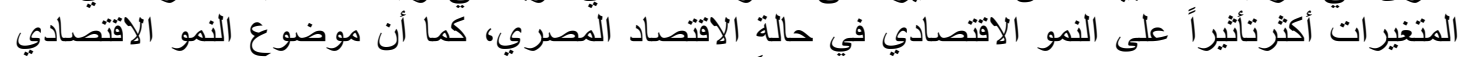

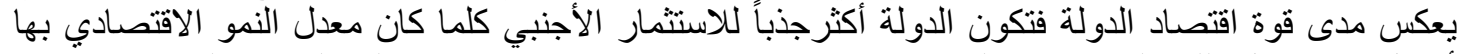

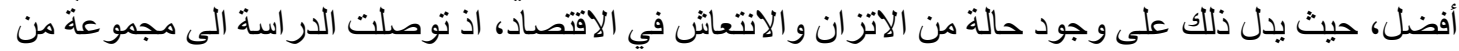

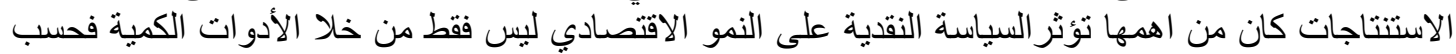




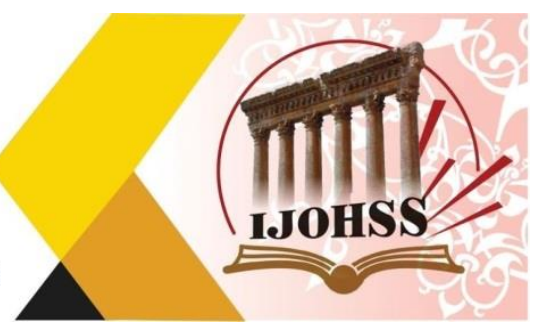

،بل و الادوات الكيفية أيضاً حيث تعد الادوات الكيفية صاحبة الاثر المباثر ، لذلك توصى الدر اسة بانه لكى تتمكن

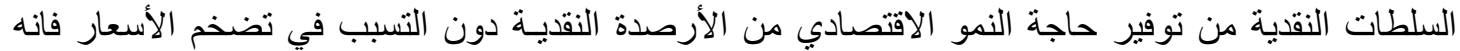

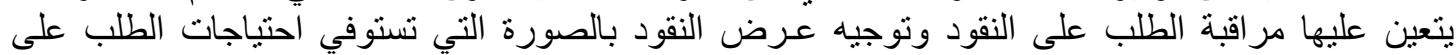

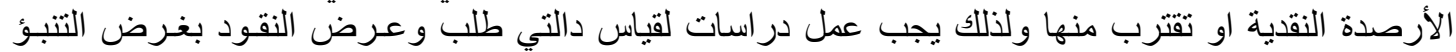

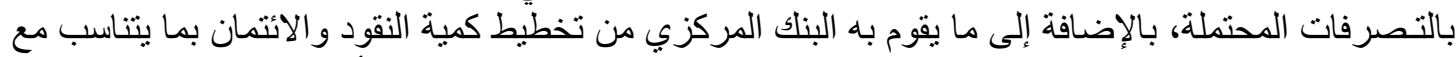

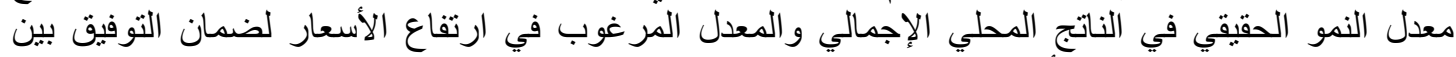
مقتضيات التنمية ومقتضيات الأسعار.

2- دراسة (Sesay Brima, Emmanuel Alpha Mansaray-Pearce, 2015) بعنوان العلاقة

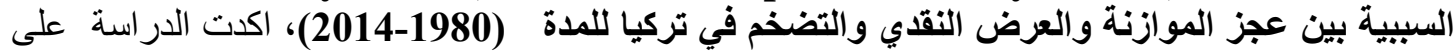

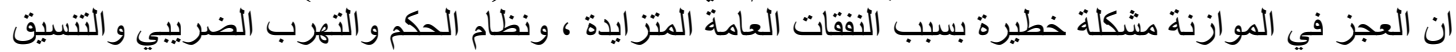

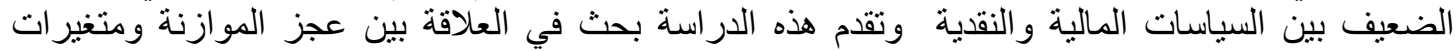

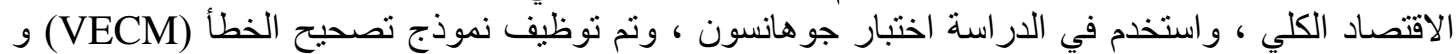

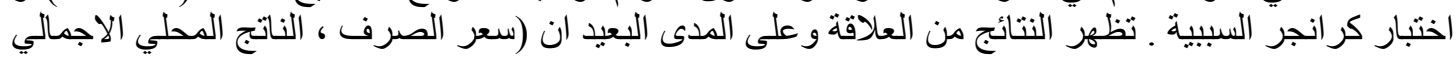

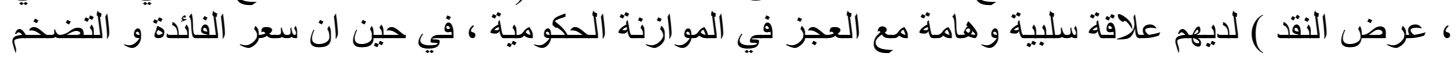

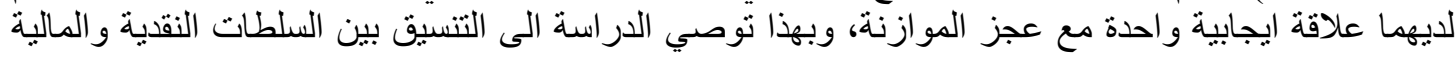

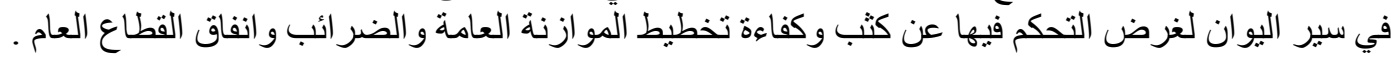

3- دراسة (صالح، علي سلمان، 2003) بعنوان عجز الموازنة والاداء الاقتصادي، تهدف الدراسة الى بيان

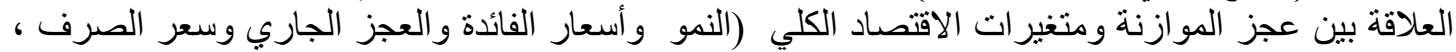

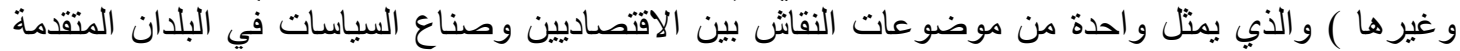

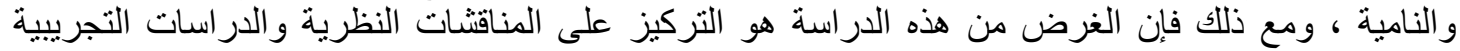

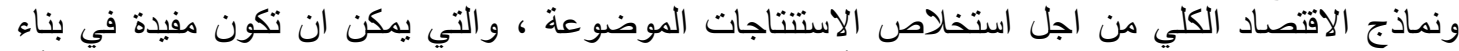

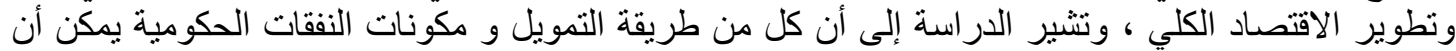

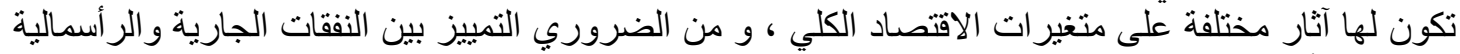

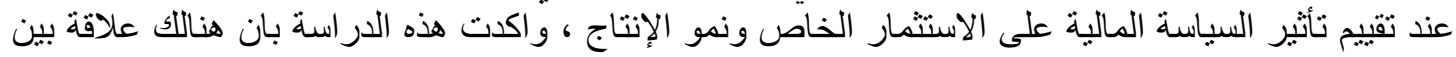

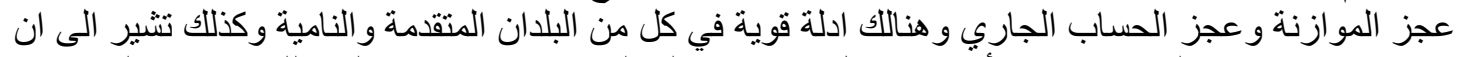

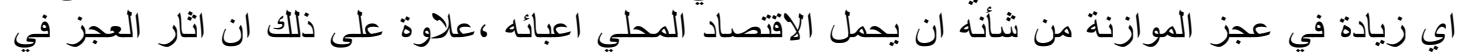

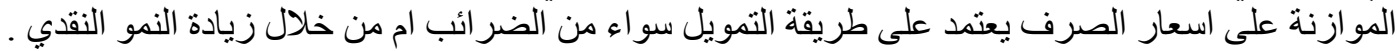

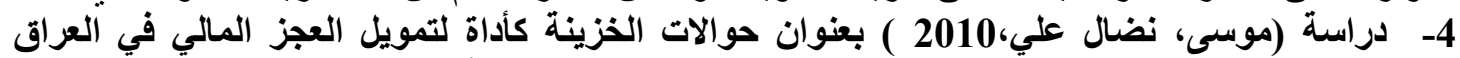

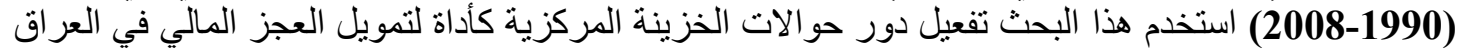

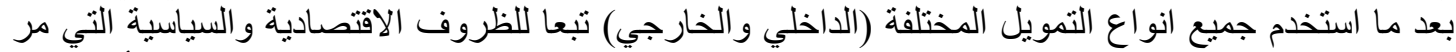

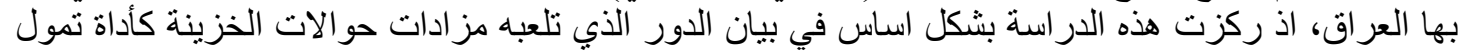

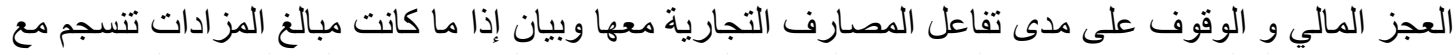

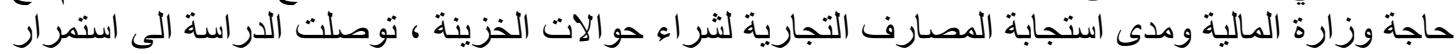

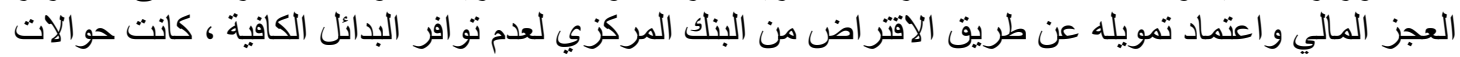

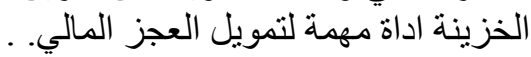




\section{المبحث الثاني \\ الاطار النظري}

1 - السياسة النقديّة

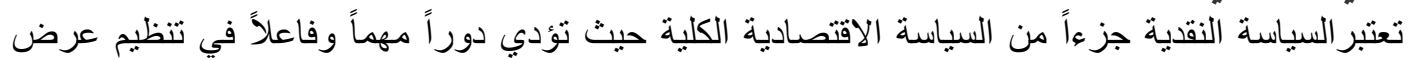

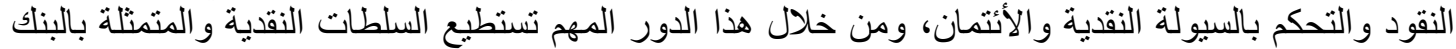

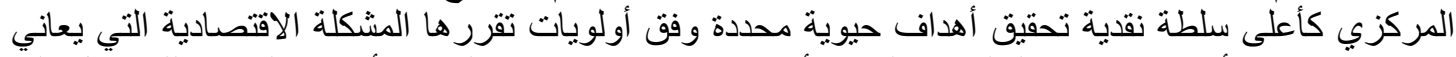

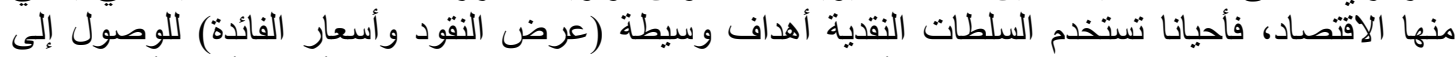

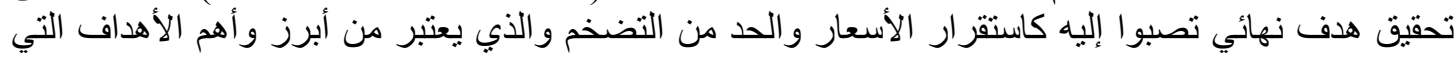

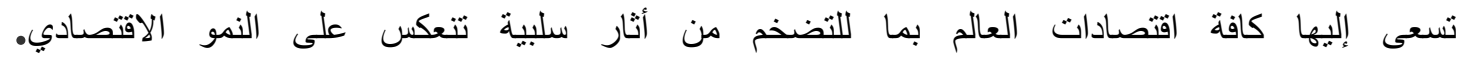

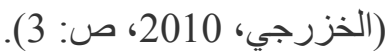

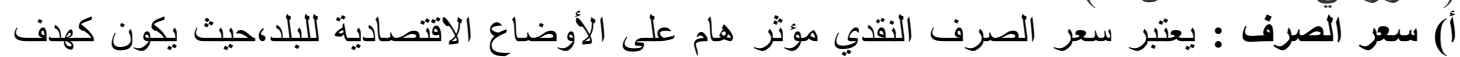

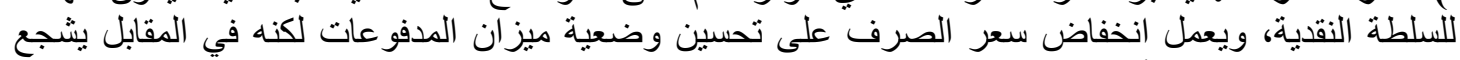

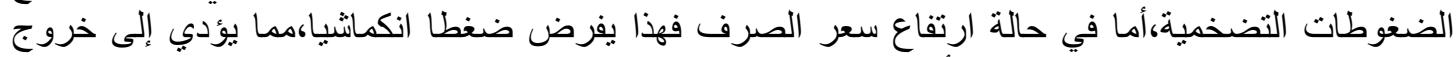

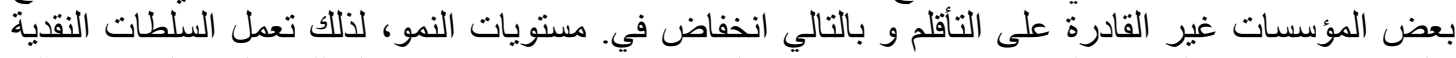

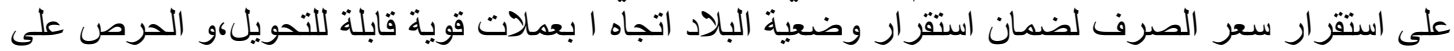

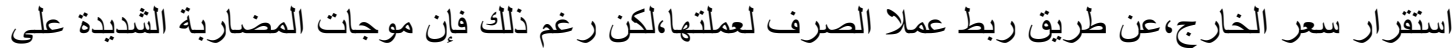

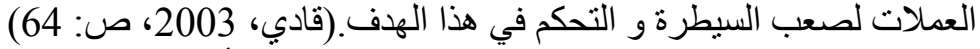

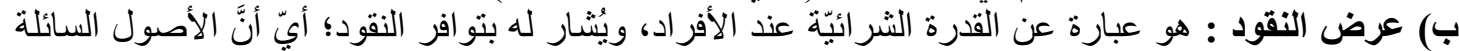

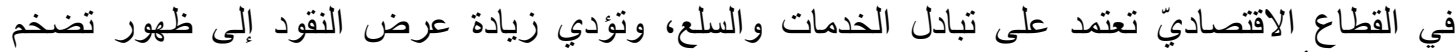

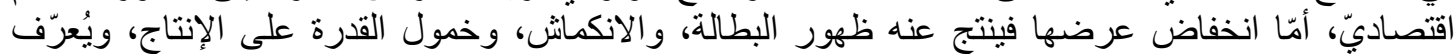

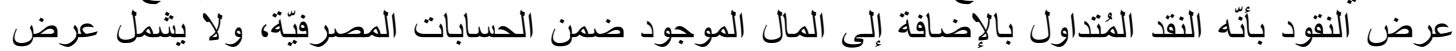

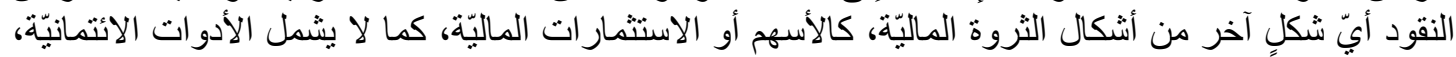

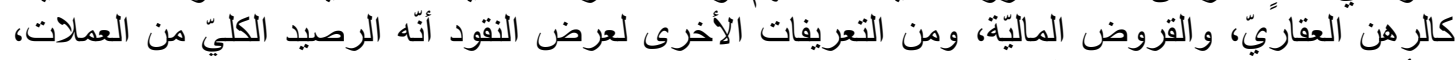

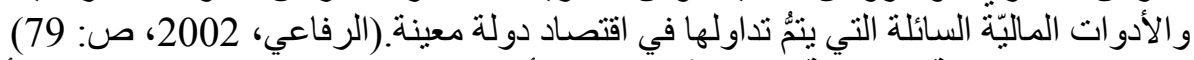

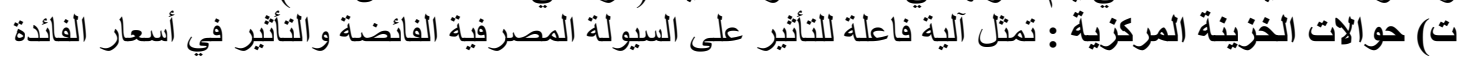

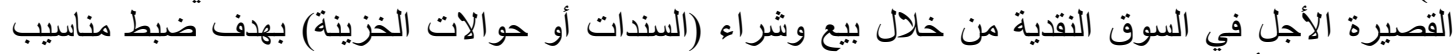

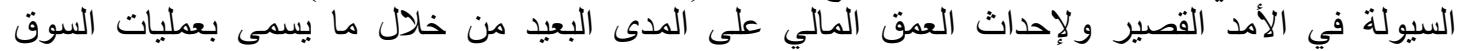

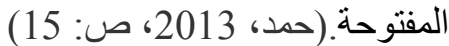
ث) التضخم: هو زيادة في النقود المتداولة بينتج عنها زيادة في الأسعار ، و انخفاض القيمة الثر ائية للنقود، وتظهر

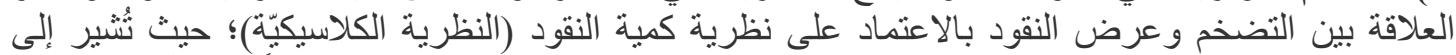

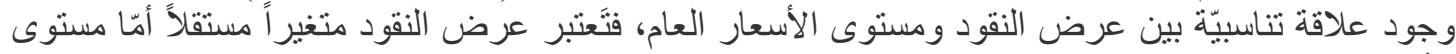

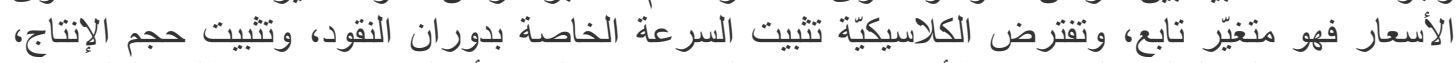

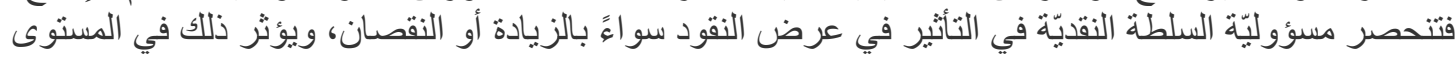

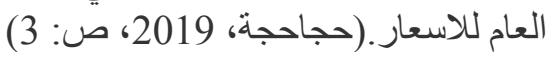

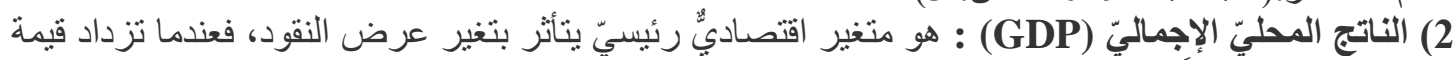

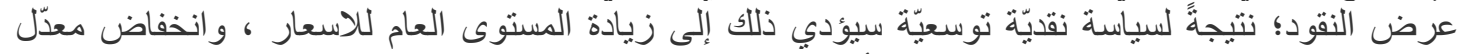

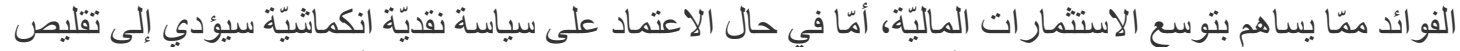

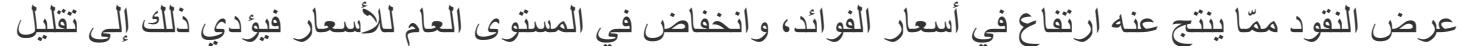

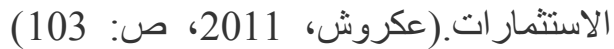


المجلة اللحولية اللملوم الآسانية والإمتصاعية International Journal of Humanities and Social Sciences website:www.ijohss.com Email:editor@ijohss.com 2020 لسبتمبر العدد (15) ISSN: 2415 - 4822 Volume (15) September 2020

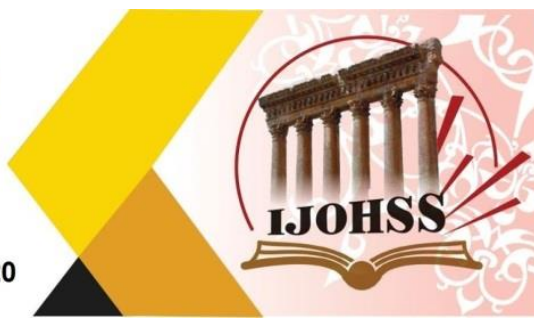

المبحث الثالث : عرض النتائج وتحليلها وفقاً لانموذج التحليل القياسي

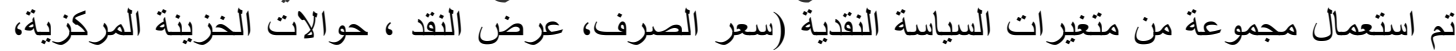

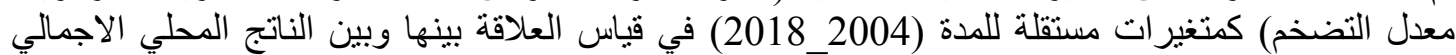
للاقتصاد العر اقي وبأستخدام البرنامج الاحصائي ( Eviews9 ) لبيان فاعلية العيانية السياسة النقدية في تنشيط الناتج المحلي الاجمالي، اذ يشمل التحليل على انموذجين وكالاتي : لإني

$$
\begin{aligned}
& \mathbf{Y = f}(\mathbf{x}) \\
& \mathbf{Y}=\mathbf{G D P} \\
& \quad \mathbf{X}=(\mathbf{E X}, \mathbf{M S}) \\
& \mathbf{G D P}=\mathbf{f}(\mathbf{E X}, \mathbf{M S})
\end{aligned}
$$

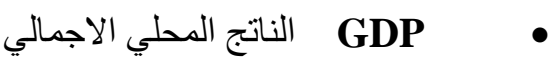

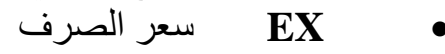

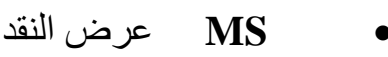

$$
\begin{aligned}
& \text { الانموذج الثاني: }
\end{aligned}
$$

\begin{tabular}{|c|c|c|c|c|c|}
\hline 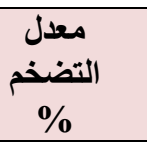 & المركزية/مليون دينار & عرض النقا مليون & سينار/دولار الصرف & مليون دينار GDP مع النفط & السنوات \\
\hline 26.8 & 4476660 & 10148626 & 1453 & $\mathbf{5 3 2 3 5 3 5 8}$ & 2004 \\
\hline 37.1 & 4434707 & 11399125 & 1475 & 73533598 & 2005 \\
\hline 53.1 & 3486137 & 15460060 & 1475 & 95587954 & 2006 \\
\hline 30.9 & 1238186 & 21721167 & 1267 & 111455813 & 2007 \\
\hline 12.7 & 1219235 & 28189934 & 1203 & 157026061 & 2008 \\
\hline 8.3 & 5197715 & 37300030 & 1182 & 130643200 & 2009 \\
\hline 2.5 & 5944472 & 51743489 & 1186 & 162064565 & 2010 \\
\hline 5.6 & 4210525 & 62473929 & 1196 & 217327107 & 2011 \\
\hline 6.1 & 6547519 & 63738571 & 1233 & 254225490 & 2012 \\
\hline 1.9 & 4255549 & 73830964 & 1232 & 273587529 & 2013 \\
\hline 2.2 & 9520019 & 72692448 & 1214 & 258900633 & 2014 \\
\hline 1.4 & 75340233 & 65435425 & 1247 & 207876191 & 2015 \\
\hline
\end{tabular}

$$
\begin{aligned}
& Y=\mathbf{f} x \\
& Y=G D P \\
& X=(I N F, \text { CTT }) \\
& \text { GDP = } \mathbf{f}(\text { INF }, \text { CTT })
\end{aligned}
$$

$$
\begin{aligned}
& \text { GDP } \\
& \text { معدل التضخم INF } \\
& \text { CTT }
\end{aligned}
$$

جدول (1) بعض متغيرات السياسة النقدية مع (GDP) للمدة (2004_2018) 
المجلة اللحولية اللملوم الآسانية والإمتصاعية International Journal of Humanities and Social Sciences websiterwww.ijohss.com Email:editor@ijohss.com العدد (15) العبتهبر 2020 ISSN: 2415 - 4822 Volume (15) September 2020

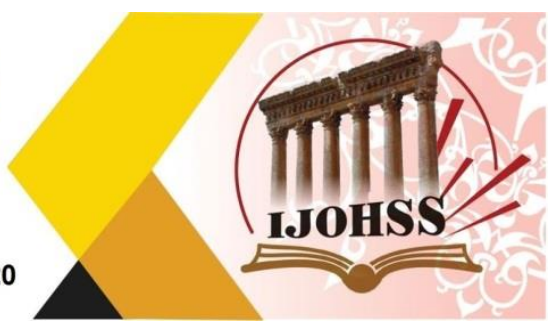

\begin{tabular}{|r|r|r|r|r|r|}
\hline $\mathbf{0 . 1}$ & $\mathbf{6 5 0 2 7 1 0 8 3}$ & $\mathbf{7 0 7 3 3 0 2 7}$ & 1275 & 196536350 & 2016 \\
\hline $\mathbf{0 . 2}$ & $\mathbf{3 2 2 9 4 6 2 4}$ & $\mathbf{7 6 9 8 6 5 7 4}$ & 1246 & $\mathbf{2 2 5 7 2 2 3 7 5}$ & 2017 \\
\hline $\mathbf{0 . 4}$ & $\mathbf{3 2 2 9 4 6 2 4}$ & $\mathbf{7 5 6 7 6 6 8 9}$ & $\mathbf{1 2 4 6}$ & $\mathbf{2 5 1 0 6 4 4 7 9}$ & $\mathbf{2 0 1 8}$ \\
\hline
\end{tabular}

المصدر :البنك المركزي العر اقي ، المديرية العامة للاحصاء والابحاث ، نشرات مختلفة.

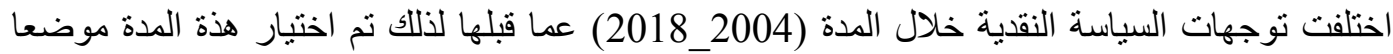
للار اسة، وبسبب قصر السلسلة الزمنية نم استخدام معادلات (Diz Approach) لتحويل البيانات السنوية الى لى فصلية وكالاتي (Diz,1971 ,p 93).

$X_{1}=Z_{t-1}+7.5 / 12\left(Z_{t}-Z_{t-1}\right)$

$X_{2}=Z_{t-1}+10.5 / 12\left(Z_{t}-Z_{t-1}\right)$

$X_{3}=Z_{t}+1.5 / 12\left(Z_{t+1}-Z_{t-1}\right)$

$X_{4}=Z_{t}+4.5 / 12\left(Z_{t+1}-Z_{t-1}\right)$

(t) ترمز الى قيمة المتغير في السنة الحالية Z

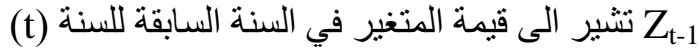

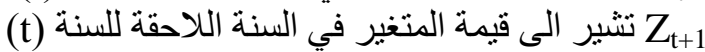

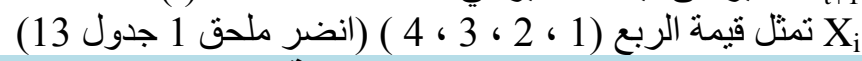
نتائج اختبار السلاسل الزمنية

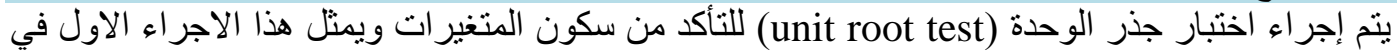

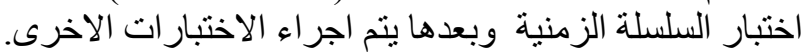

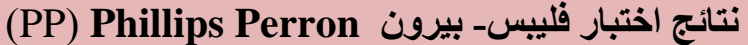
$(2$

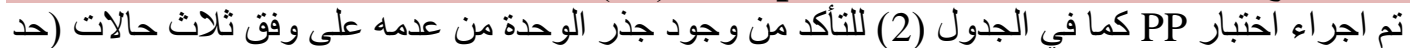

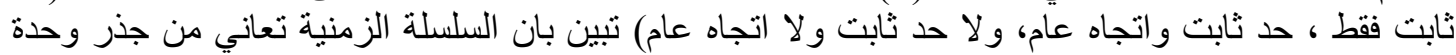

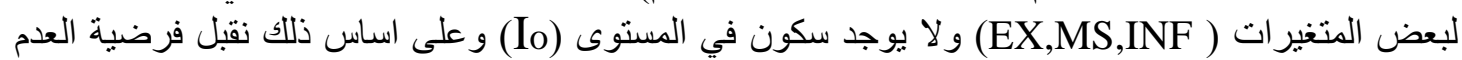
و التي تنص على وجود جذر وحدة ، وبعد اخذ الفرق الاول ( I1 (I1)o) للسلسة الزمنية الربع سنوية للمدة

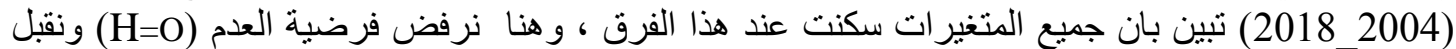

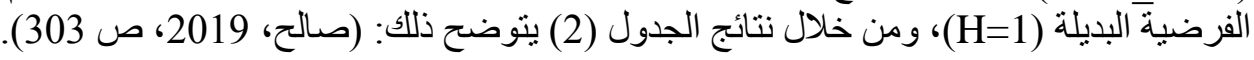

جدول (2) اختبار فيلبس بيرون لجذر الوحدة (P P unit root test)

\begin{tabular}{|c|c|c|c|c|c|c|c|c|c|}
\hline \multirow[t]{3}{*}{ المتغير } & \multicolumn{3}{|r|}{ المستوى } & \multicolumn{3}{|c|}{ الفروق الاولى } & \multicolumn{3}{|r|}{ الفرق الثاني } \\
\hline & حل ثنابت & واتجاه عام & 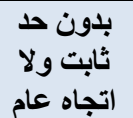 & هـ ثابت & واتجاه عام & 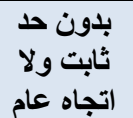 & حلث ثابت & واتجاة عابت & 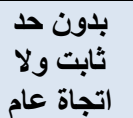 \\
\hline & Prob & Prob & Prob & Prob & Prob & Prob & Prob & Prob & Prob \\
\hline GDP & 0.006 & 0.006 & 0.376 & 0.000 & 0.000 & 0.000 & 0.0001 & 0.0001 & 0.000 \\
\hline EX & 0.044 & 0.164 & $\overline{0.323}$ & 0.000 & $\overline{0.000}$ & 0.000 & 0.0001 & 0.0001 & 0.000 \\
\hline MS & 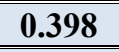 & 0.234 & 0.480 & 0.000 & 0.000 & 0.000 & 0.0001 & $\begin{array}{l}0.0001 \\
\end{array}$ & 0.000 \\
\hline INF & 0.451 & 0.520 & 0.097 & 0.000 & 0.000 & 0.000 & 0.0001 & 0.0001 & 0.000 \\
\hline CTT & 0.0003 & 0.000 & 0.0001 & 0.0001 & 0.0001 & 0.000 & 0.0001 & 0.0001 & 0.000 \\
\hline
\end{tabular}

المصدر : اعداد الباحث بالاعتماد على مخرجات برنامج 


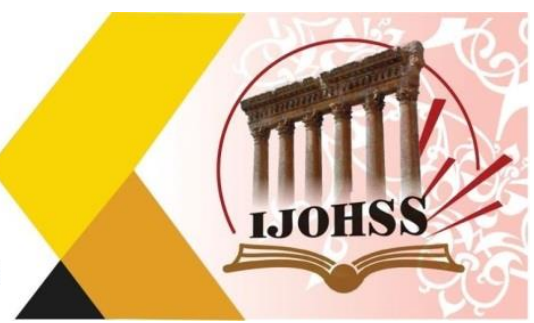

بعد اجر اء اختبار السكون (unit root test) و وفقا لنتائج اختبار فليبس بيرون (p p) ، تلبين ان بعض الإن

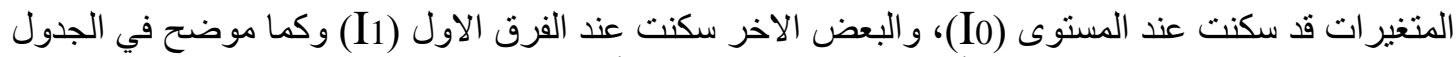

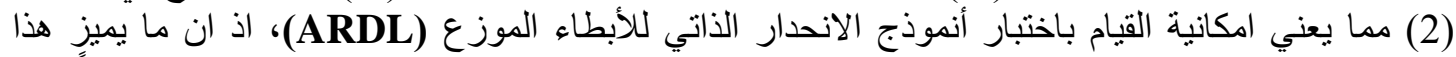

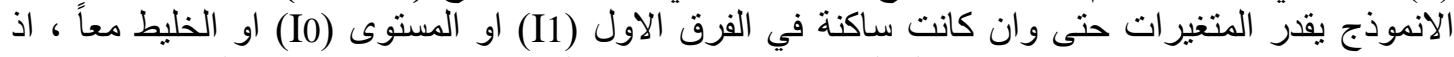
نلاحظ ان الانموذج يقوم باختيار عدد التخلفات (lag) وبطريقة تلقائية وهذه احد ميز الته (الكبيسي، 2010،

3) نتائج اختبار (ARDL) للانموذج الاول (ARL) (145)

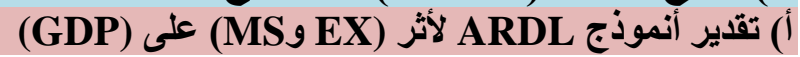

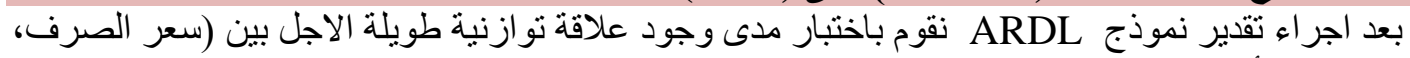

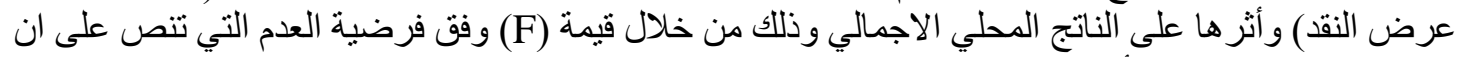

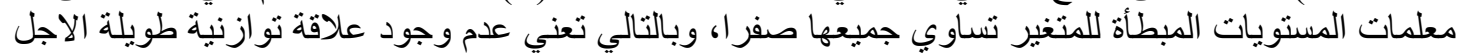

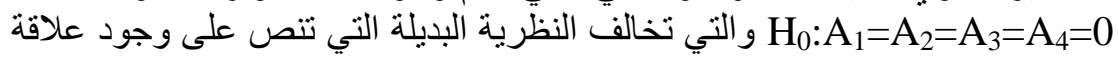

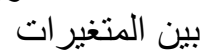

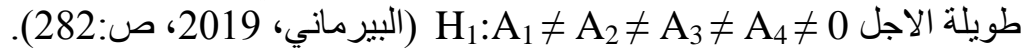

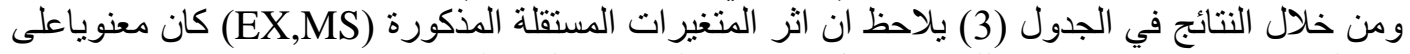

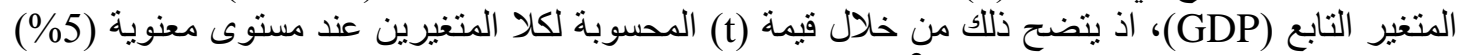
كما توضح قيمة معامل التحديد (RDe

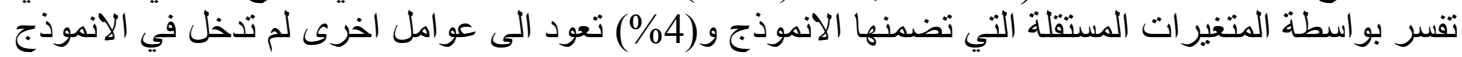

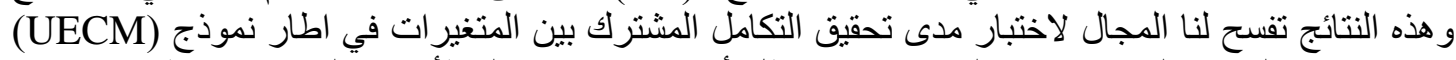

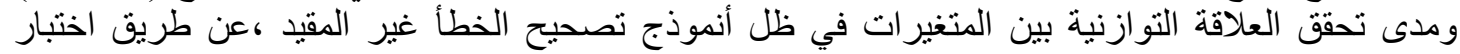

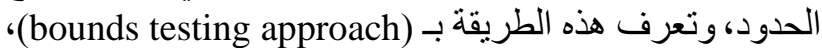

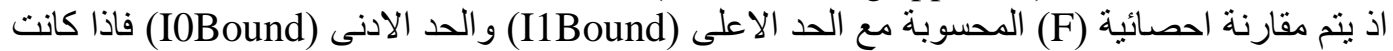

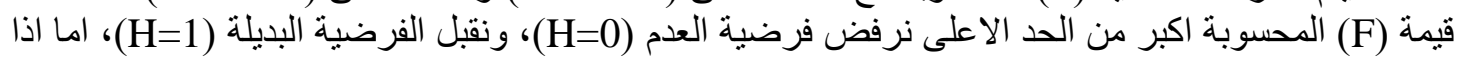

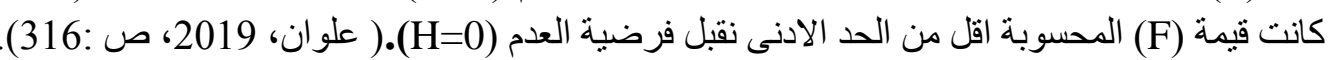
جدول (3) تقدير أنموذج ARDL لأثر (EX و (MS) على (GDP) (

\begin{tabular}{|c|c|c|c|c|}
\hline Variable & Coefficient & Std. Error & t- Statistic & Prob.* \\
\hline GDP(-1) & 0.544221 & 0.085632 & 6.355342 & 0.0000 \\
\hline EX & -88728.35 & 100012.7 & -0.887171 & 0.3803 \\
\hline MS & 1.298466 & 1.535797 & 0.845467 & 0.4029 \\
\hline $\mathrm{C}$ & $1.87 \mathrm{E}+08$ & 57036364 & 3.282709 & 0.0021 \\
\hline R-squared & 0.961067 & \multicolumn{2}{|c|}{ Mean dependent var } & $2.81 \mathrm{E}+08$ \\
\hline Adjusted R-squared & 0.947440 & \multicolumn{2}{|c|}{ S.D. dependent var } & $1.06 \mathrm{E}+08$ \\
\hline S.E. of regression & 24243999 & \multicolumn{2}{|c|}{ Akaike info criterion } & 37.07224 \\
\hline Sum squared resid & $2.35 E+16$ & \multicolumn{2}{|c|}{ Schwarz criterion } & 37.61969 \\
\hline Log likelihood & -1004.487 & \multicolumn{2}{|c|}{ Hannan-Quinn criter. } & 37.28394 \\
\hline F-statistic & 70.52872 & \multicolumn{2}{|c|}{ Durbin-Watson stat } & 1.387419 \\
\hline $\operatorname{Prob}(F-$ statistic) & 0.000000 & & & \\
\hline
\end{tabular}

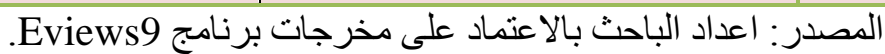

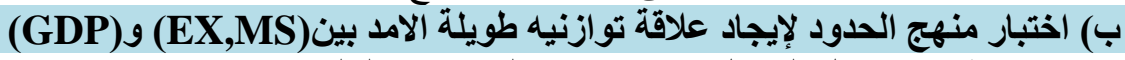

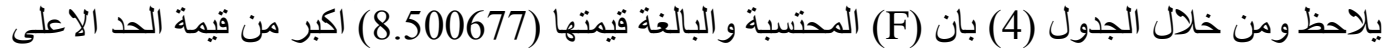

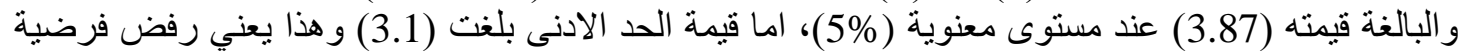

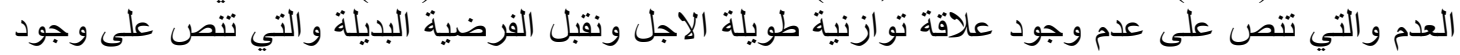
علاقة نو ازنية طويلة الاجل بين متغير ات الدراسة، اذ بلغت قيمة معامل التحديد ( R-squared 94\%) و قيمة التية 
المجلة الدولية اللهلور الأسانسية والإمتماعية International Journal of Humanities and Social Sciences website:www.ijohss.com Email:editor@ijohss.com العدد (15) ل 2020 ISSN: 2415 - 4822 Volume (15) September 2020

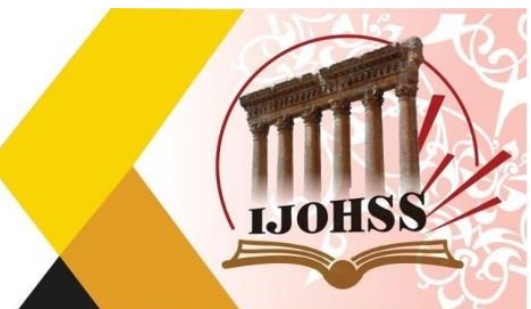

معامل التحديد المرجح (Adjusted R-squared 92\% ) و هذا يثير الى قوة العلاقة التفسيرية بين المتغير ات.

جدول(4) اختبار منهج الحدود لإيجاد علاقة توازنيه طويلة الامد بين(EX,MS) و(GDP) للمدة (-2018) 2004

\begin{tabular}{|c|c|c|}
\hline Test Statistic & Value & K \\
\hline F.Statistic & 8.500677 & 2 \\
\hline Significance & Critical Value Bounds & \\
\hline $10 \%$ & (I0) Bound & (I1) Bound \\
\hline $5 \%$ & 2.63 & 3.35 \\
\hline $2.5 \%$ & 3.1 & 3.87 \\
\hline $1 \%$ & 3.55 & 4.38 \\
\hline R-squared $(0.945827)$ & 4.13 & 5 \\
\hline
\end{tabular}

المصدر : اعداد الباحث بالاعتماد على مخرجات برنامج Eviews.

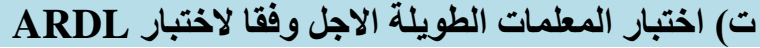

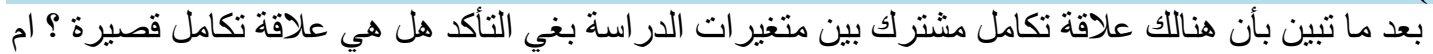

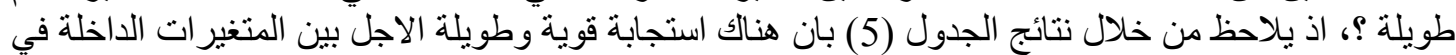

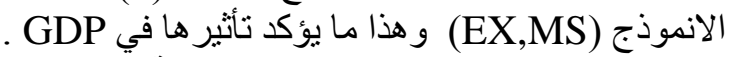

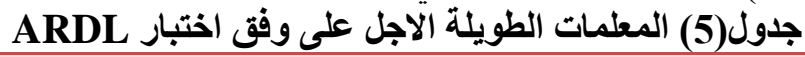

\begin{tabular}{|l|l|l|l|l|}
\hline \multicolumn{5}{|c|}{ Long Run Coefficients } \\
\hline Variable. & Coefficient & Std. Error & t-Statistic & Prob \\
\hline EX & -554158.113 & 203220.675 & -2.726879 & 0.0094 \\
\hline MS & 0.680848 & 1.538757 & 0.442466 & 0.6605 \\
\hline C & 939053141.662 & 315005798.447 & 2.981066 & 0.0049 \\
\hline
\end{tabular}

المصدر : اعداد الباحث بالاعتماد على مخرجات برنامج Eviews9.

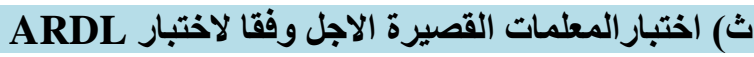

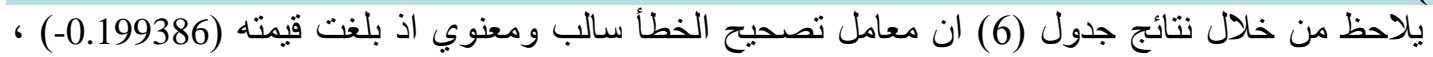

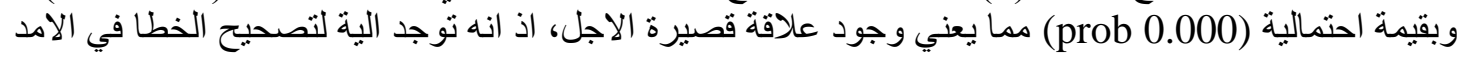

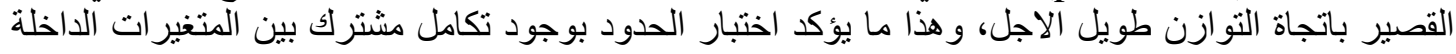

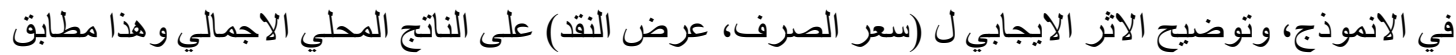
ل اللنظرية الاقتصادية.

جدول (6) المعلمات القصيرة الاجل على وفق اختبار ARDL

\begin{tabular}{|l|l|l|l|l|}
\hline \multicolumn{5}{|c|}{ Cointegrating Form } \\
\hline Variable & Coefficient & Std. Error & t-Statistic & Prob. \\
\hline D(EX) & -88728.349 & 89604.367 & -0.990223 & 0.3280 \\
\hline D(MS) & 1.298466 & 1.380462 & 0.940602 & 0.3526 \\
\hline CointEq(-1) & -0.199386 & 0.032979 & -6.045900 & 0.0000 \\
\hline
\end{tabular}

المصدر : اعداد الباحث بالاعتماد على مخرجات برنامج Eviews9. 


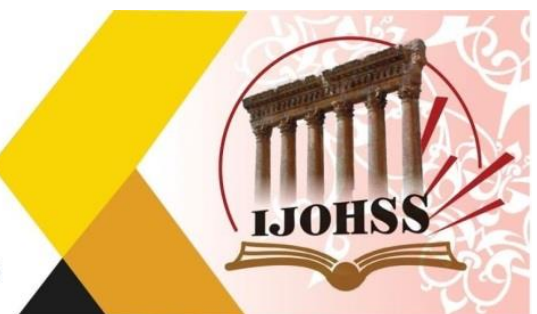

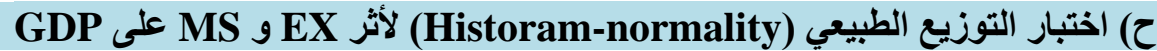

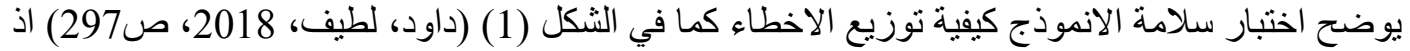

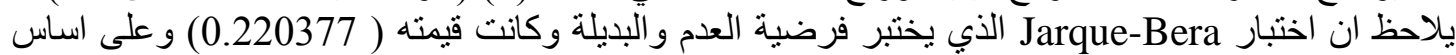

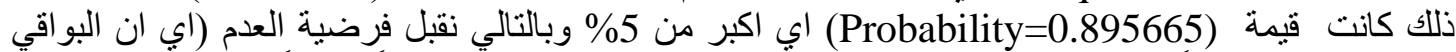

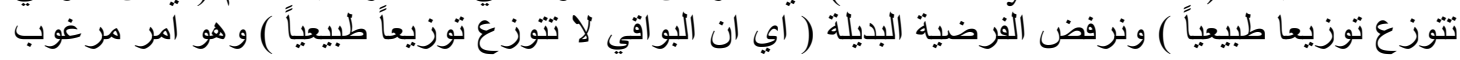

\section{شكل (1) اختبار التوزيع الطبيعي (Historam-normality) لأثر EX و MS على GDP للمدة} (2018_2004)

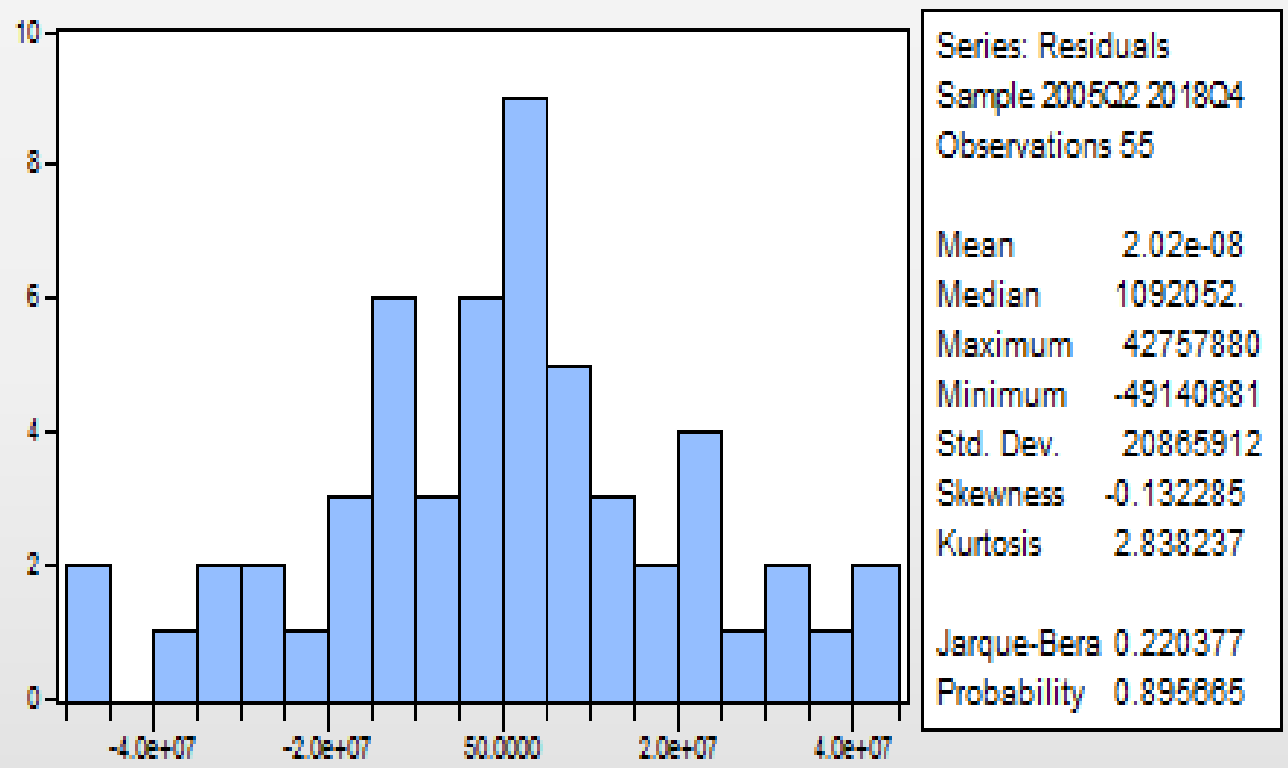

المصدر: اعداد الباحث بالاعتماد على مخرجات برنامج Eviews9.

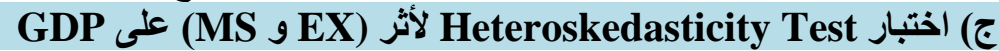

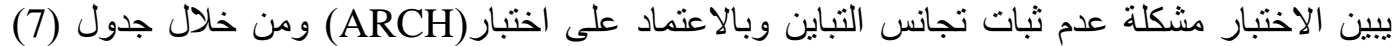

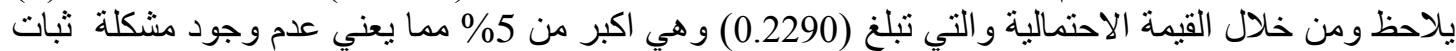

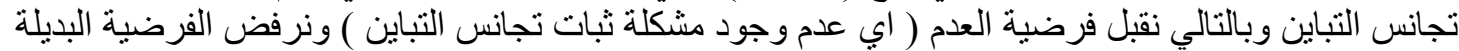
( اي وجود مشكلة ثبات تجانس التباين ) ).

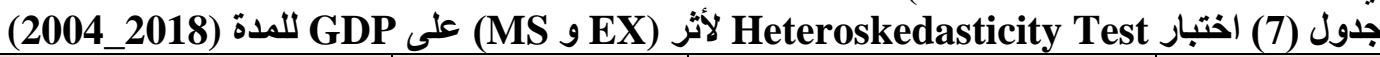

\begin{tabular}{|l|l|l|l|}
\hline F-statistic & 1.481494 & Prob. F(2,42) & 0.2290 \\
\hline Obs*R-squared & 1.495857 & Prob. Chi-Square(2) & 0.2213 \\
\hline R-squared & 0.027701 & Mean dependent var & $4.35 \mathrm{E}+14$ \\
\hline Adjusted R-squared & 0.009003 & S.D. dependent var & $5.88 \mathrm{E}+14$ \\
\hline S.E. of regression & $5.85 \mathrm{E}+14$ & Akaike info criterion & 70.87979 \\
\hline Sum squared resid & $1.78 \mathrm{E}+31$ & Schwarz criterion & 70.95346 \\
\hline Log likelihood & -1911.754 & Hannan-Quinn criter. & 70.90820 \\
\hline F- statistic & 1.481494 & Durbin-Watson stat & 1.934120 \\
\hline Prob(F-statistic) & & \\
\hline
\end{tabular}

المصدر : اعداد الباحث بالاعتماد على مخرجات برنامج Eviews9. 


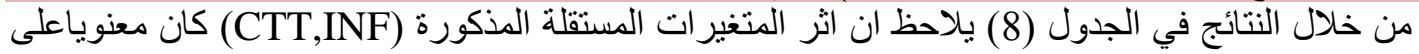

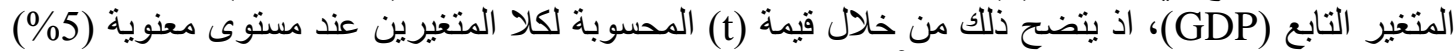

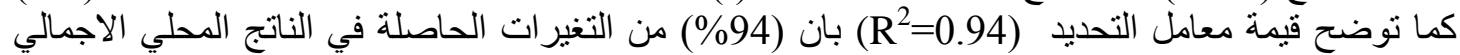

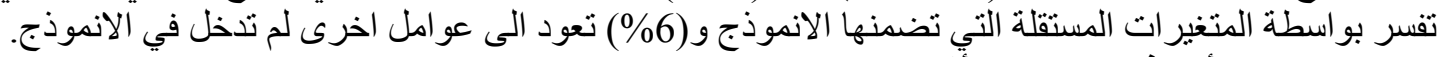

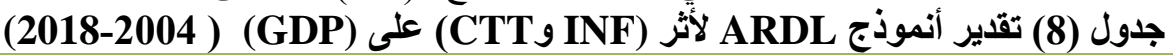

\begin{tabular}{|c|c|c|c|c|}
\hline Variable & Coefficient & Std. Error & t- Statistic & Prob. \\
\hline $\operatorname{GDP}(-1)$ & 0.833430 & 0.083498 & 9.981465 & 0.0000 \\
\hline INF & -315294.5 & 310163.9 & -1.016541 & 0.3149 \\
\hline CTT & 0.033555 & 0.016747 & 2.003611 & 0.0513 \\
\hline $\mathrm{C}$ & 34799742 & 18124732 & 1.920014 & 0.0614 \\
\hline R-squared & 0.942966 & \multicolumn{2}{|c|}{ Mean dependent var } & $2.81 \mathrm{E}+08$ \\
\hline Adjusted R-squared & 0.930003 & \multicolumn{2}{|c|}{ S.D. dependent var } & $1.06 \mathrm{E}+08$ \\
\hline S.E. of regression & 27977943 & \multicolumn{2}{|c|}{ Akaike info criterion } & 37.30859 \\
\hline Sum squared resid & $3.44 E+16$ & \multicolumn{2}{|c|}{ Schwarz criterion } & 37.71005 \\
\hline Log likelihood & -1014.986 & \multicolumn{2}{|c|}{ Hannan-Quinn criter. } & 37.46384 \\
\hline F-statistic & 72.74671 & \multicolumn{2}{|c|}{ Durbin-Watson stat } & 1.257052 \\
\hline Prob(F- statistic) & 0.000000 & & & \\
\hline
\end{tabular}

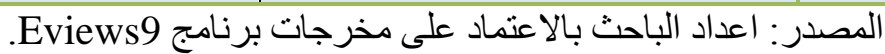

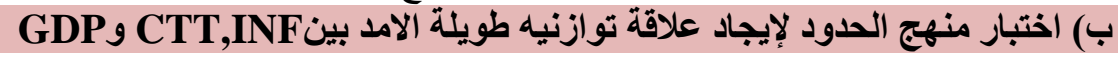

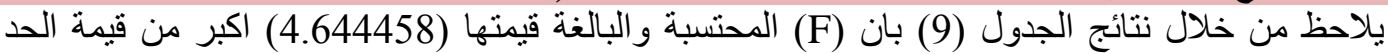

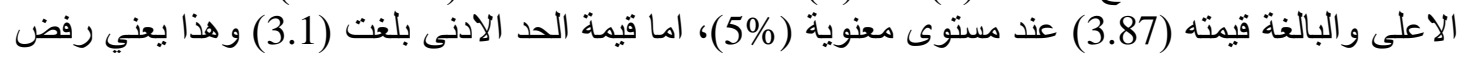

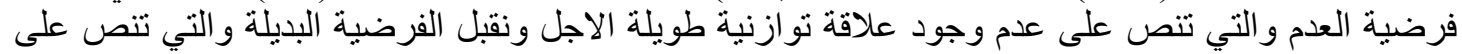

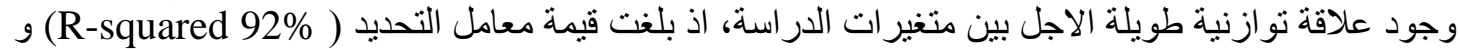
قيمة معامل التحديد المرجح (Adjusted R-squared 90\% ) و هذا يشير الى قوة العلاقة التفسيرية بين

جدول(9) اختبار منهج الحدود لإيجاد علاقة توازنيه طويلة الامد بين(CTT,INF) و(GDP) للمدة (2018-2004)

\begin{tabular}{|c|c|c|}
\hline Test Statistic & Value & K \\
\hline F.Statistic & 4.644458 & 2 \\
\hline Significance & Critical Value Bounds & \\
\hline $10 \%$ & (I0) Bound & (I1) Bound \\
\hline $5 \%$ & 2.63 & 3.35 \\
\hline $2.5 \%$ & 3.1 & 3.87 \\
\hline $1 \%$ & 3.55 & 4.38 \\
\hline R-squared (0.920641) & $4.13 \quad$ AdjustedR-squared (0.902605) \\
\hline
\end{tabular}

المصدر : اعداد الباحث بالاعتماد على مخرجات برنامج Eviews9. 


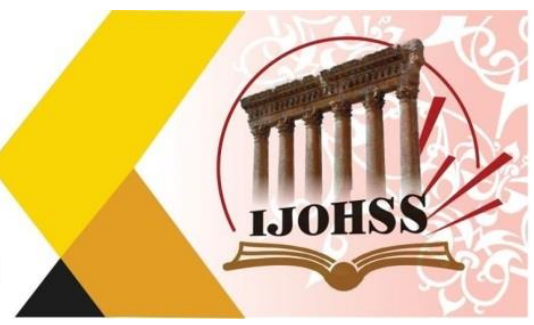

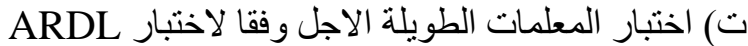

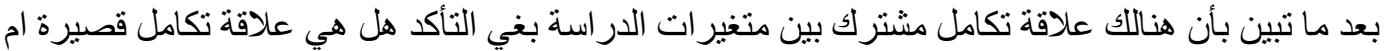

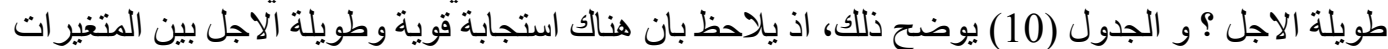

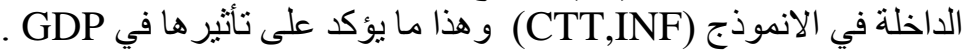
جدول(10) المعلمات الطويلة الاجل على وفق ناتير اختبار ARDL

\begin{tabular}{|l|l|l|l|l|}
\hline \multicolumn{5}{|l|}{ Long Run Coefficients } \\
\hline Variable. & Coefficient & Std. Error & t-Statistic & Prob \\
\hline INF & -3025919.255 & 2281534.189 & -1.326265 & 0.1916 \\
\hline CTT & -0.614765 & 0.410041 & -1.499277 & 0.1409 \\
\hline C & 333977324.005 & 52978871.393 & 6.303972 & 0.0000 \\
\hline
\end{tabular}

المصدر : اعداد الباحث بالاعنماد على مخرجات برنامج

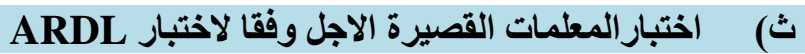

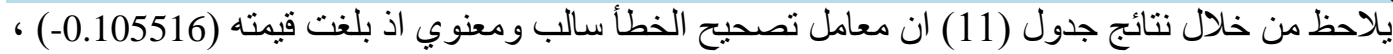

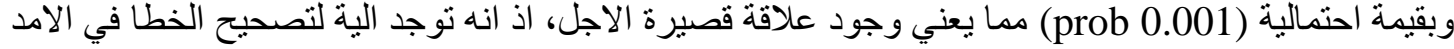

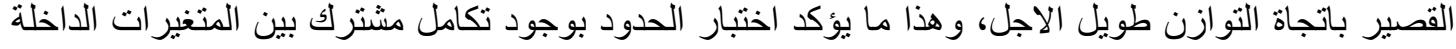
في الانموذج، وتوضيح الاثر الايجابي ل (التضخم، حوالاتئ الات الخزينة المركزية) على الناتج المحلي الاجمالي وهذا مطابق للنظرية الاقتصلدية.

جدول (11) المعلمات القصيرة الاجل على وفق اختبار FormL

\begin{tabular}{|l|l|l|l|l|}
\hline \multicolumn{5}{|c|}{ Cointegrating Form } \\
\hline Variable & Coefficient & Std. Error & t-Statistic & Prob. \\
\hline D(EX) & 79210.565 & 725541.259 & 0.109174 & 0.9136 \\
\hline D(MS) & 0.033121 & 0.015879 & 2.085900 & 0.0428 \\
\hline CointEq(-1) & -0.105516 & 0.023690 & -4.454003 & 0.0001 \\
\hline
\end{tabular}

المصدر : اعداد الباحث بالاعتماد على مخرجات برنامج

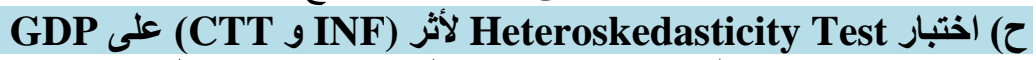

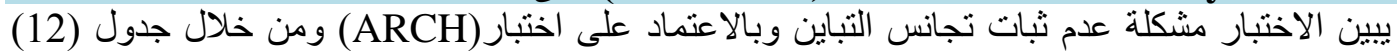

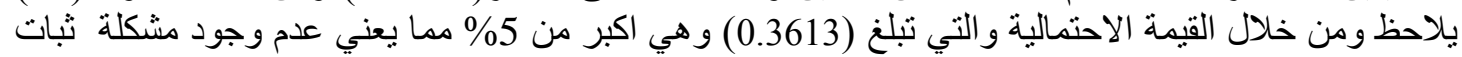

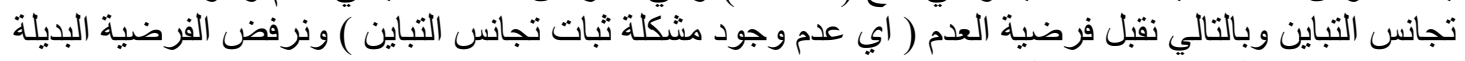

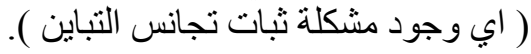
جدول (12) اختبار Heteroskedasticity Test لأثر (INF) و GTT) على GDP للمدة (CT) (2012_204)

\begin{tabular}{|l|l|l|r|}
\hline F-statistic & 0.848263 & Prob. F(2,42) & 0.3613 \\
\hline Obs*R-squared & 0.866749 & Prob. Chi-Square(2) & 0.3519 \\
\hline R-squared & 0.016051 & Mean dependent var & $6.34 \mathrm{E}+14$ \\
\hline Adjusted R-squared & -0.002871 & S.D. dependent var & $1.17 \mathrm{E}+15$ \\
\hline S.E. of regression & $1.18 \mathrm{E}+15$ & Akaike info criterion & 72.27521 \\
\hline Sum squared resid & $7.19 \mathrm{E}+31$ & Schwarz criterion & 72.34887 \\
\hline Log likelihood & -1949.431 & Hannan-Quinn criter. & 72.30362 \\
\hline F- statistic & 0.848263 & Durbin-Watson stat & 2.006273 \\
\hline Prob(F-statistic) & 0.361297 & & \\
\hline
\end{tabular}




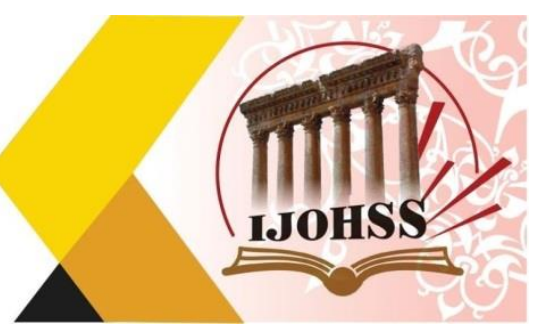

ج) اختبار سكون انموذج ARDL المقدر

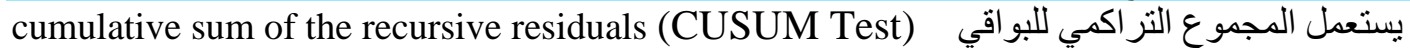

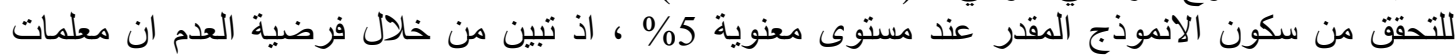

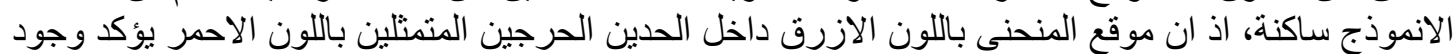

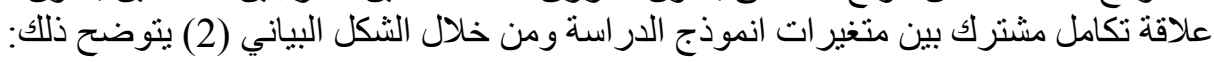

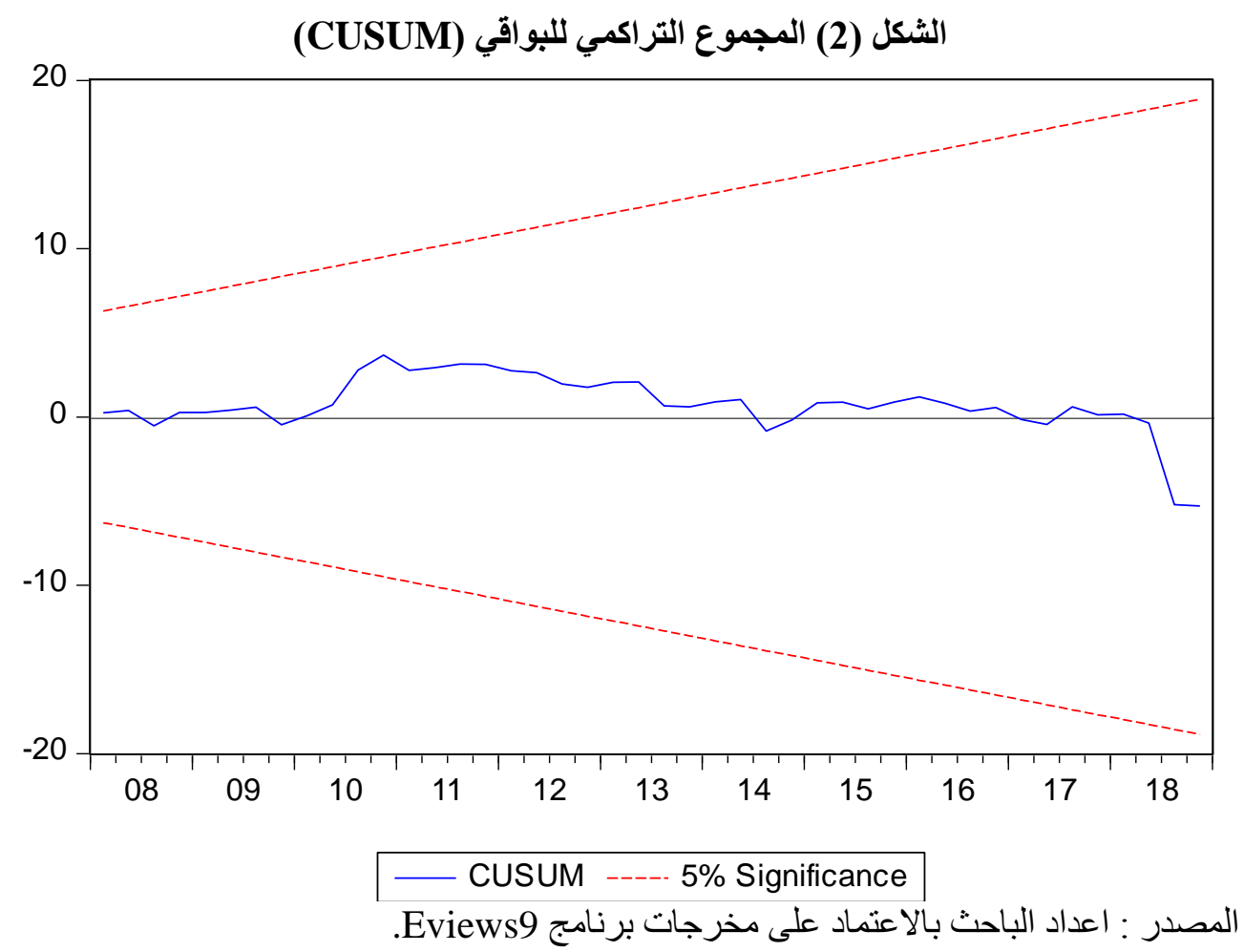

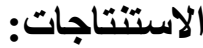

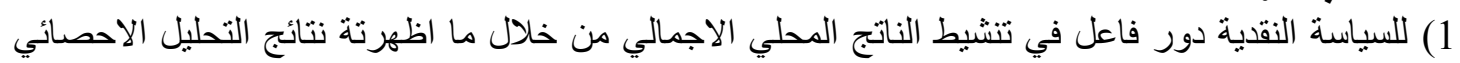

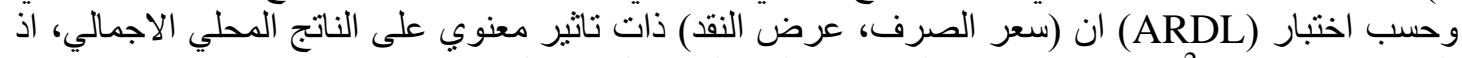

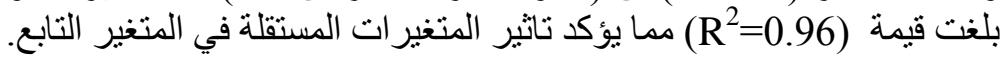

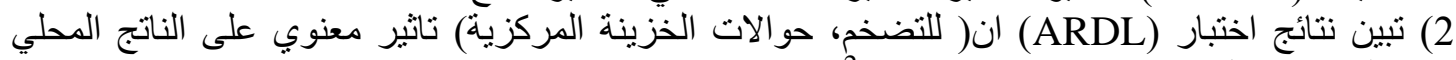

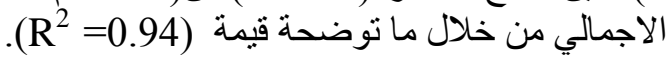

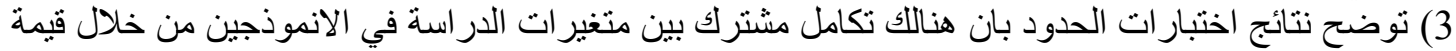

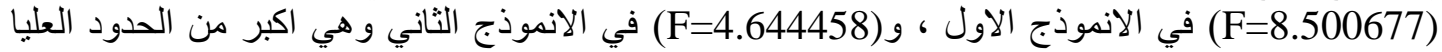

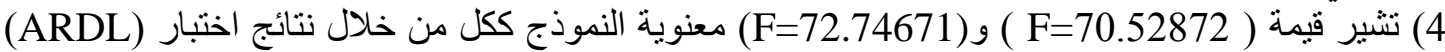

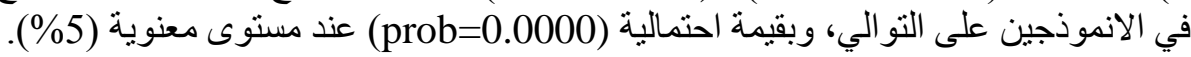

التوصيات:

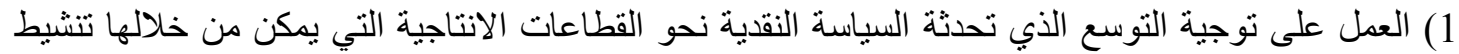
الناتج المحلي الاجمالي عن طريق زيادة الاستثمار ات فيها باعتبار ان الاستثمار احد مكونات GDP مما يؤدي النيات

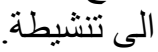




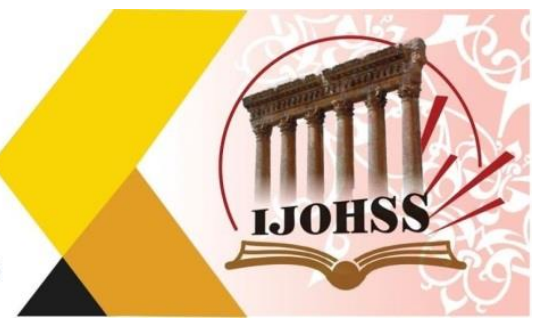

2) احداث عجز مقصود من قبل السياسة النقدية في بعض القطاعات المنتجة (صناعة، زر اعة) التي يمكن من

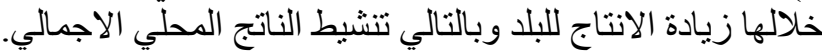

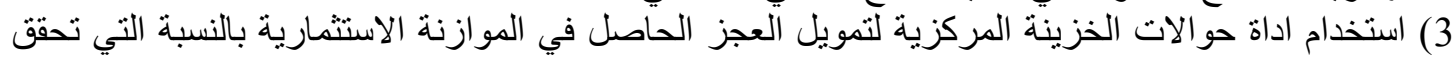
زيادة في الناتج مما يؤدي الى تنتشيط الناتج المحلي التئي الاجمالي.

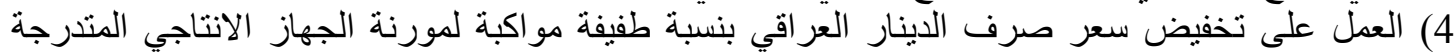

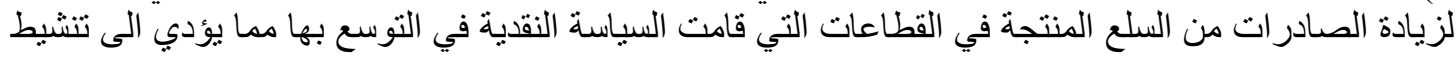
باعتبار الصادر ات احد مكوناتة.

1. المصادر الخزرجي، ثريا عبد الرحيم، 2010، السياسة النقدية في العراق بين تراكمات الماضي وتحديات

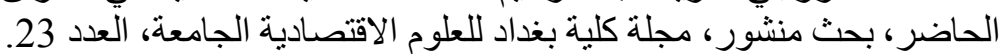

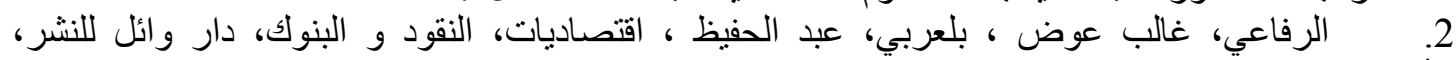

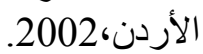

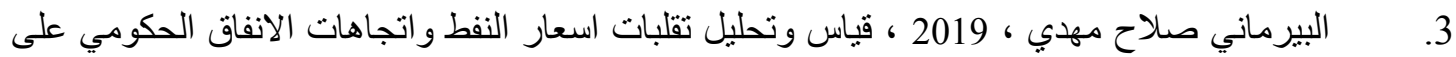

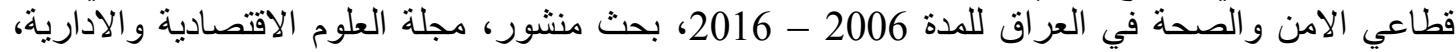

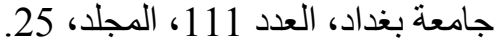

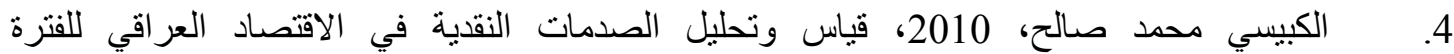

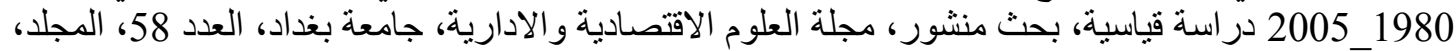
6.

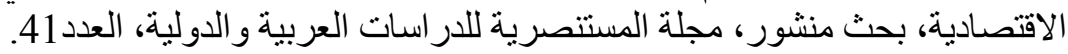

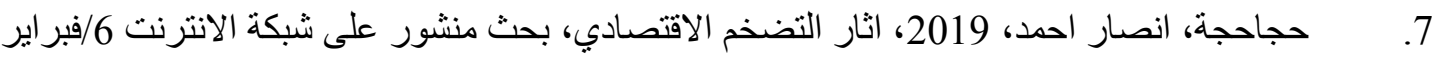
https:// mawdoo3.com 8. داود سمير سهام، لطيف مقداد غضبان، 2018 ، قياس ناثير هيمنة النمط الاستهلاكي للانفاق الحكومي

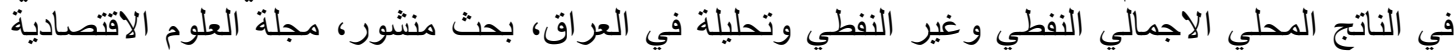
و الادارية، جامعةٌ بغداد، العدد 102، الإلجي المجلد، 24.

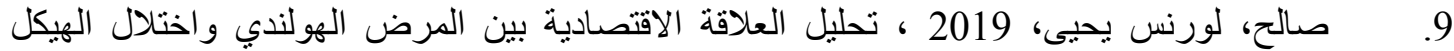

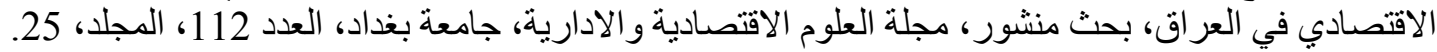

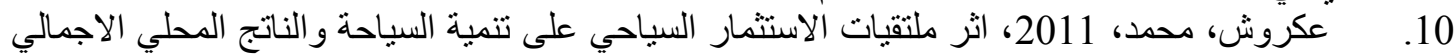

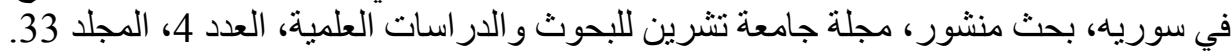

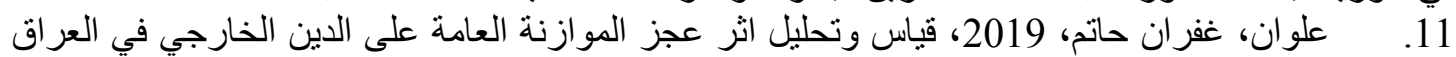

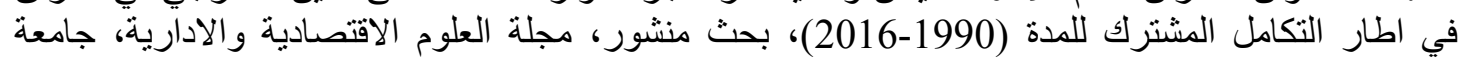

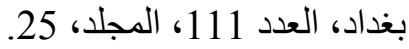
12. قادي، عبد المجيد ، المدخل الى السياسات الاقتصادية الكلية،ديوان المطبو عات الجامعية، الجزائر، .2003

13. Diz,Amoney and Prices in Argentina, 1935-1962,Journal of Money and Banking,USA 1971. 
المجلة اللدوالية اللملوم الآنسانية والإمتماعية International Journal of Humanities and Social Sciences website:www.ijohss.com Email:editor@ijohss.com ISSN: 2415 - 4822
(15) العدد (15) September 2020

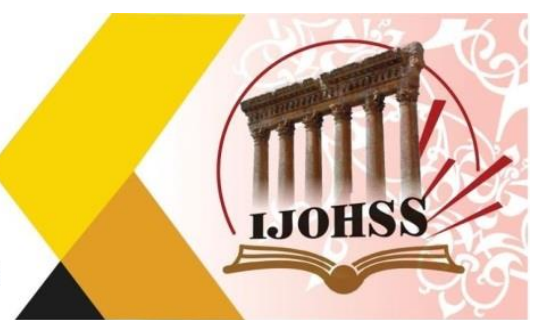

\section{References}

1) Akroush, Muhammad, 2011, The Impact of Tourism Investment Forums on the Development of Tourism and GDP in Syria, published research, Tishreen University Journal for Research and Scientific Studies, No 4, Vol 33.

2) Al-Birmani Salah Mahdi, 2019, Measuring and analyzing oil price fluctuations and trends in government spending on the security and health sectors in Iraq for the period 2006-2016, published research, Journal of Economic and Administrative Sciences, University of Baghdad, No 111, Vol 25.

3) Al-Khazraji, Thuraya Abdul-Rahim, 2010, Monetary Policy in Iraq Between the Accumulations of the Past and the Challenges of the Present, Published Research, Journal of Baghdad College of Economic Sciences, No 23.

4) Al-Kubaisi Muhammad Salih, 2010, Measuring and Analyzing Monetary Shocks in the Iraqi Economy for the Period 1980-2005, Standard Study, Published Research, Journal of Economic and Administrative Sciences, University of Baghdad, No 58, Vol 16.

5) Al-Rifai, Ghaleb Awad, Belarbi, Abdel Hafeez, Economics, Money and Banks, Wael Publishing House, Jordan, 2002.

6) Alwan, Ghufran Hatim, 2019, Measurement and Analysis of the Impact of the Public Budget Deficit on the External Debt in Iraq in the Context of Joint Integration for the Period (1990-2016), Published Research, Journal of Economic and Administrative Sciences, University of Baghdad, No 111, Vol 25.

7) Dawood Samir Siham, Latif Miqdad Ghadhban, 2018, Measuring the influence of the dominance of the consumption pattern of government spending on oil and non-oil GDP and analyzing it in Iraq, published research, Journal of Economic and Administrative Sciences, Baghdad University, No 102, Vol. 24.

8) Diz,Amoney and Prices in Argentina, 1935-1962,Journal of Money and Banking,USA 1971.

9) Hajjah, Ansar Ahmed, 2019, The Effects of Economic Inflation, Research published on the Internet https// : February 6, mawdoo3.com

10) Hamad, Abdul Rahim Maktouf, 2013, Bonds and Treasury Remittances in Iraq and their Impact on Economic Development, published research, AlMustansiriya Journal for Arab and International Studies, No 41.

11) Kady, Abdel-Majid, Introduction to Macroeconomic Policies, University Press Office, Algeria, 2003.

12) Saleh, Lawrence Yahya, 2019, Analysis of the economic relationship between Dutch disease and the disruption of the economic structure in Iraq, published research, Journal of Economic and Administrative Sciences, University of Baghdad, No. 112, Vol. 25 .

13) The Central Bank of Iraq, General Directorate of Statistics and Research, various publications. 
المجلة المولية اللملوم الآسانية والإمتماعية International Journal of Humanities and Social Sciences website:www.ijohss.com Email:editor@ijohss.com

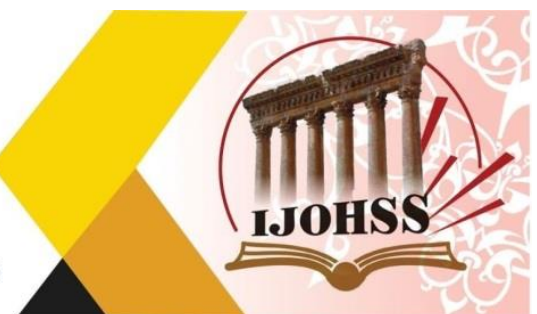

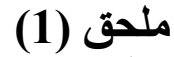

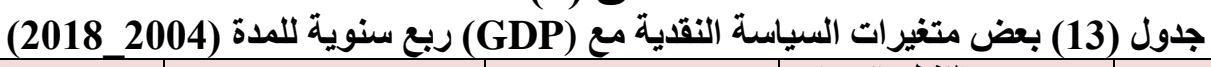

\begin{tabular}{|c|c|c|c|c|c|}
\hline $\begin{array}{l}\text { التضخم } \\
\text { \% }\end{array}$ & سينار/ دولارف & حوالات الخزينة المركزية & مليون دينار & 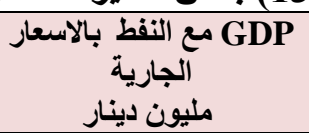 & السنوات \\
\hline 24.13 & 1433 & 4975165.87 & 6523340.33 & 64330028.5 & 2004(1) Q \\
\hline $\mathbf{0}$ & 1455 & 5972177.62 & 7511462 & 56933581.5 & 2004(2) Q \\
\hline 30.1 & 1463 & 4975165.87 & 8419791.33 & 123070732.5 & 2004(3) Q \\
\hline 29.36 & 1463 & 5972177.62 & 9036204 & 115674285.5 & 2004(4) Q \\
\hline 43.2 & 1462 & 6969189.37 & 10561101 & 93496857.25 & $2005(1) Q$ \\
\hline 36.4 & 1472 & 7966201.12 & 10893188 & 80188017.75 & $2005(2) Q$ \\
\hline 36.96 & 1479 & 4316135.75 & 10684919.66 & 162467132.3 & $2005(3) Q$ \\
\hline 32.73 & 1477 & 4078993.25 & 10980389.33 & 149158292.8 & $2005(4) Q$ \\
\hline 39.9 & 1481 & 3841850.75 & 11969080.66 & 123163053.3 & $2006(1) Q$ \\
\hline 51.2 & 1484 & 3604708.25 & 11973776 & 104779653.8 & 2006(2) Q \\
\hline 65.93 & 1487 & 3205143.12 & 13505420.66 & 197852067.3 & 2006(3) Q \\
\hline 56.43 & 1448 & 2643155.37 & 14309216 & 179468667.8 & $2006(4) Q$ \\
\hline 46.7 & 1302 & 2081167.62 & 15905566.33 & 147301295.8 & $2007(1) Q$ \\
\hline 41.83 & 1275 & 1519179.87 & 15995704.33 & 123404307.3 & 2007(2)Q \\
\hline 28.43 & 1254 & 1235817.12 & 17870826.66 & 256533379.8 & $2007(3) Q$ \\
\hline 13.53 & 1233 & 1231079.37 & 20785308.88 & 232636391.3 & 2007(4) Q \\
\hline 5 & 1224 & 1226341.62 & 21980881 & 198821990.9 & $2008(1) Q$ \\
\hline 1.26 & 1211 & 1221603.87 & 25329815.33 & 170958037.6 & 2008(2) Q \\
\hline 2.1- & 1195 & 1716545.2 & 25329815.33 & 273737284.4 & 2008(3) Q \\
\hline 7.03 & 1182 & 27111654.1 & 27131367 & 245873331.1 & $2008(4) Q$ \\
\hline $0.7-$ & 1178 & 3705785.2 & 29703804.33 & 189527972.9 & 2009(1) Q \\
\hline 3.53- & 1182 & 4700405.33 & 29565727 & 150271457.6 & 2009(2) Q \\
\hline $1.5-$ & 1184 & 5291059.62 & 32728162.66 & 273079507.4 & 2009(3) $Q$ \\
\hline $5.23-$ & 1184 & 5477748.87 & 36131866 & 233822992.1 & 2009(4) Q \\
\hline 2.7 & 1185 & 5664438.12 & 39375752.33 & 211055765 & 2010(1) Q \\
\hline 2.06 & 1185 & $\mathbf{5 8 5 1 1 2 7 . 3 7}$ & 45079880.33 & 178394965 & 2010(2) Q \\
\hline 1.66 & 1185 & 5727728.62 & 47841314.33 & 363061272 & 2010(3) $Q$ \\
\hline 3.33 & 1185 & 5294241.87 & 50197610 & 330400472 & 2010(4) Q \\
\hline 5.56 & 1185 & 4860755.12 & 50793702.66 & 278101318.9 & 2011(1) Q \\
\hline 6.16 & 1193 & 4427268.37 & 51329479.33 & 237585177.6 & 2011(2) Q \\
\hline 5.5 & 1199 & 4502649.25 & 55327471.33 & 451294526.4 & 2011(3) Q \\
\hline 5.2 & 1206 & 5086897.75 & 60035958.33 & 410778385.1 & 2011(4) Q \\
\hline 6.46 & 1227 & 5671146.25 & 60719379.33 & 335723155.1 & 2012(1) Q \\
\hline 7.23 & 1251 & 6255394.75 & 61558726.33 & 281391378.4 & 2012(2) Q \\
\hline 6.36 & 1243 & 6261022.75 & 60874847.33 & 500647130.6 & 2012(3) Q \\
\hline 4.3 & 1210 & 5688030.25 & 62350513.33 & 446315353.9 & 2012(4) Q \\
\hline 2.1 & 1237 & 5115037.75 & 65070603.33 & 368922087.8 & 2013(1) Q \\
\hline
\end{tabular}


المجلة اللحولية اللملوم الآسانية والإمتصاعية International Journal of Humanities and Social Sciences website:www.ijohss.com Email:editor@ijohss.com
لسبتهبر 2020 Volume (15)
العدد (15)

September 2020

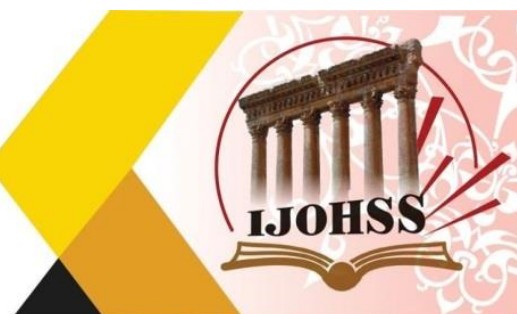

\begin{tabular}{|c|c|c|c|c|c|}
\hline 1.53 & 1258 & 4542045.25 & 70296036 & 305365715.3 & $2013(2) Q$ \\
\hline 0.9 & 1212 & 4913607.75 & 70584483.33 & 500709975.8 & 2013(3) Q \\
\hline 2.96 & 1220 & 6229725.25 & 72469285.33 & 437153603.3 & 2013 (4) Q \\
\hline 3 & 1222 & 7545842.75 & 73229087 & 361495956.4 & 2014(1) Q \\
\hline 1.7 & 1218 & 8861960.25 & 72864588.66 & 293099074.1 & 2014(2) Q \\
\hline 2.36 & 1211 & 18405604.5 & 70890003 & 432578382.9 & 2014(3) Q \\
\hline 1.83 & 1204 & 36176775.5 & 71366151.33 & 364181500.6 & 2014(4) Q \\
\hline 0.03 & 1244 & 50657652.75 & 69383361 & 304963928.4 & 2015(1) Q \\
\hline 1.46 & 1304 & 67112706.25 & 71935649.66 & 240238770.1 & 2015(2) Q \\
\hline 2.43 & 1223 & 155434116 & 68729972 & 372049961.9 & $2015(3) Q$ \\
\hline 1.63 & 1218 & 315621882 & 66382409.33 & 307324803.6 & 2015(4) Q \\
\hline 0.8 & 1245 & 434672014.25 & 67365266.66 & 274489921.6 & $2016(1) Q$ \\
\hline 1.56 & 1276 & 578404726.75 & 70165035.33 & 222520873.9 & 2016(2) Q \\
\hline 0.03 & 1281 & 434672014.25 & 71118564 & 396274201.1 & 2016(3) Q \\
\hline 5.- & 1299 & $\mathbf{5 7 8 4 0 4 7 2 6 . 7 5}$ & 70613580 & 344305153.4 & $2016(4) Q$ \\
\hline 0.23 & 1724 & 276146280.1 & 103511459.1 & 299423506.3 & 2017(1) Q \\
\hline 0.21 & 1405 & 11357850.94 & 85828202.38 & 250289418.8 & $2017(2) Q$ \\
\hline 0.58 & 2332 & 166946378.3 & 143821634.6 & 452219810.3 & 2017(3) Q \\
\hline 0.56 & 2013 & 1792624081 & 126138377.9 & 403085722.8 & 2017(4) Q \\
\hline 0.47 & 1713 & 444051080 & 104546654.3 & 335710369.6 & 2018(1) Q \\
\hline 0.42 & 1401 & 363314521 & 85300010.75 & 279279775.9 & 2018(2) Q \\
\hline 0.37 & 1090 & 282577961 & 66053367.25 & 222849182.1 & 2018(3) Q \\
\hline 0.32 & 778 & 201841402 & 46806723.75 & 166418588.4 & 2018(4) Q \\
\hline
\end{tabular}

المصدر اعداد الباحث بالاعتماد على بيانات الجدول (1) و استخدام معادلات Diz. 